\title{
Transporte público de buses versus congestión y contaminación en Lima y Callao*
}

\author{
Manuel Martínez Espinal ${ }^{* *}$
}

\begin{abstract}
RESUMEN
Se mide la influencia del transporte público de buses sobre la congestión vehicular y sobre la contaminación ambiental de Lima y Callao. Se modela el efecto de los flujos de buses sobre la red de transporte mediante un software de asignación dinámica de tráfico Dynasmart. Se construye la base de datos sobre el Plan Maestro 2005-2025. Para ello, se verifica la red de transporte por Google Earth, y se utilizan mediciones de campo sobre capacidad, velocidad, y funciones volumen-demora que describen la congestión vial. Se calculan los flujos de buses con el software Emme3. Se construyen las matrices origen-destino por cada hora a partir de la matriz origendestino de 24 horas del Plan Maestro. En el caso de la congestión, se mide una influencia exacta y otra máxima de los flujos de buses, y también el caso de segregar un carril de vía para un sistema de buses rápidos, un tema frecuente de discusión. En el caso de la contaminación, se utiliza la influencia exacta de los flujos de buses para medir consumo de combustible y emisión de contaminantes para la alternativa de reducción de buses de transporte público y otras cinco alternativas más, entre ellas la implementación de un sistema de buses rápidos con buses a gas. Se obtiene que, reducir la flota de buses a la mitad, incrementa la velocidad de la red de transporte entre 2 y $7 \%$ diario y entre 9 y $14 \%$ a las 11 am, y que produce un $46 \%$ de los beneficios de promover combustibles limpios e inspección como mantenimiento; es decir, que esta medida es poco efectiva en reducir la congestión y que su prioridad debiera reducirse en aliviar la contaminación.
\end{abstract}

Palabras clave: transporte público, congestión vehicular, externalidades, países en desarrollo Códigos JEL:

\section{Public bus services versus congestion and pollution in Lima and Callao}

\begin{abstract}
This study measures the influence of public bus services on traffic congestion and environmental pollution in Lima and Callao. The effect of the flow of buses on the transportation network is measured by way of a dynamic traffic assignment program, Dynasmart. The database is

\footnotetext{
* Fondos del Concytec hicieron posible la adquisición de software y de construcción de parte de la base de datos necesarios para este artículo científico (contrato 194-2005-CONCYTEC-OAJ). Asimismo, el profesor Bladikas del New Jersey Institute of Technology ha revisado parte crucial de la base de datos del modelo Dynasmart. Agradezco muy especialmente al Comité Editorial de la revista Economía y a los árbitros porque sus observaciones han sido muy útiles para producir este artículo.

** Economista, Econ. Ph.D., M.Sc., Colegiado CEL 4047, Profesional independiente.
} 
constructed on the basis of the 2005-2025 Master Plan. To this end, the transportation network is verified using Google Earth, and field measurements of capacity, speed, and volume-delay functions to describe traffic congestion are used. The flow of buses is calculated using the Emme3 program. Origin-destination matrices are calculated for each hour based on the 24-hour origindestination matrix from the Master Plan. In the case of congestion, both the exact and the maximum influence of the flow of buses are measured, and the case of segregating a traffic lane for a Rapid Bus System - a much-discussed topic - is also considered. In the case of pollution, the exact influence of the flow of buses is used to measure fuel consumption and pollutant emissions for the option of reducing public bus services as well as a further five options, including the implementation of a Rapid Bus System featuring gas-powered buses. It is found that halving the size of the bus fleet increases the speed of the transportation network by between 2 and $7 \%$ per day, and between 9 and $14 \%$ until 11 am, and yields $46 \%$ of the benefits of promoting clean fuels and inspection and maintenance; that is, that this measure is of limited worth in reducing congestion, and should not be considered a priority means of alleviating pollution.

Keywords: Public Transportation, Traffic Congestion, Externalities, Developing Countries. JEL Codes:

\section{INTRODUCCIÓN}

Las reformas del transporte público de Lima y Callao se han basado sobre la premisa de que los flujos de buses causan la congestión y la contaminación de la ciudad y que por ello, la flota de buses debe reducirse. Este artículo científico va a: I) medir la congestión originada por los flujos ${ }^{1}$ de buses sobre la red de transporte, y II) analizar alternativas para reducir la contaminación, incluyendo la de reducir el número de buses.

\section{EL TRANSPORTE PÚBLICO DE BUSES Y LA CONGESTIÓN VEHICULAR}

\section{El PROBLEMA DEL TRANSPORTE PÚBLICO DE BUSES}

\section{El transporte público de buses de Lima y Callao}

El transporte público de buses es vital para las actividades económicas de Lima y Callao; sirve al 77\% de sus viajes motorizados transportando 9,32 millones de pasajeros por día (Ministerio de Transportes y Comunicaciones, 2005). El crecimiento de la población ha creado necesidades urgentes de transporte que se han satisfecho a la medida de su baja productividad y de sus escasos recursos, con un sistema de buses ${ }^{2}$ de muchos vehículos, frecuentemente de tamaño mediano o pequeño, intensivo en mano de obra, bajo costo,

\footnotetext{
1 Por flujo se entiende el número de vehículos que circula por una avenida por dirección por hora. Puede referirse a toda la avenida o a un solo carril.

2 La definición de "modo de transporte público de buses" establece que esta tecnología admite todos los tamaños incluyendo combis, cústers, buses estándares de 12 metros de largo y articulados; excepto sistemas de buses rápidos, y autos-colectivos que conforman otros modos de transporte.
} 
mediana velocidad, y alta flexibilidad. A partir de 1995 se generan recursos de conocimientos técnicos, estudios, y proyectos así como parte del financiamiento para llevar adelante soluciones apropiadas al transporte urbano. Se pueden mencionar las soluciones de los trenes eléctricos, los corredores de buses rápidos tipo Metropolitano, las nuevas vías expresas de Javier Prado, Línea Amarilla, así como bypasses y nuevos puentes, y la administración público-privada de vías importantes, además de las ciclo-vías como transporte alternativo.

Desde 2010 se ha iniciado un boom de la inversión pública en este sector productivo de transporte urbano. El proceso de motorización, o de compras masivas de autos y de oferta abundante de taxis ha ańadido la urgencia de la congestión a la necesidad de la inversión público-privada en infraestructura de transporte urbano. Importantes decisiones necesitan estudiar cuidadosamente las medidas a tomarse en el transporte público y evitar el desperdicio de recursos públicos y privados.

Una solución muy frecuentemente priorizada desde 2001 ha sido la de reducir el número de buses de transporte público con el fin de combatir la congestión vehicular y de aliviar la contaminación ambiental ${ }^{3}$. Sin embargo, hasta ahora no se han medido ni su efectividad para reducir la congestión ni su prioridad para aliviar la contaminación. Este artículo científico cumple con realizar tales mediciones especiales. A continuación se presenta una breve revisión de la literatura sobre el problema del transporte público de buses en países del tercer mundo de América, Asia, y África.

\section{El transporte público de buses y la externalidad de la congestión}

En la década de 1980 el problema era la falta de vehículos, pues las compañías de transporte público no tenían los suficientes buses para satisfacer una población que crecía rápidamente; no tenían fondos y la tarifa era baja. Así pues, transportistas privados oportunistas entraban al mercado usando vehículos tipo combi para abastecer la demanda insatisfecha (Jacobs et al., 1982). Costos laborales bajos, vehículos pequeños, y operaciones flexibles para atender la demanda, les dieron gran éxito comercial a los transportistas privados (Armstrong-Wright et al., 1987). Posteriormente, las políticas de privatización y desregulación permitieron la entrada legal de los transportistas privados pero, incrementaron tanto el número de buses que generaron gran congestión y contaminación (Gwilliam et al., 1996). Al llegar los años 2000 el problema se había transformado en la congestión creada por la abundancia de buses.

Para remediar ello, se recomendó la re-regulación para reducir el número de buses mediante subastas públicas (Estache et al., 2004), aprovechando que había una sobreoferta que podía ser eliminada ${ }^{4}$. Siguiendo a Estache, la congestión y la contaminación

\footnotetext{
3 Este artículo científico mencionará otras políticas de transporte público, aunque una discusión formal está más allá del alcance de este artículo.

4 Este punto de sobreoferta contrasta nítidamente contra las políticas de transporte público de países desarrollados cuya literatura difiere en que supone que los flujos de buses usualmente no provocan congestión grave y establece que generalmente se debe favorecer al transporte público, y por ello, no corresponde incluir esta literatura en este artículo científico porque responde a otra realidad.
} 
eran fallas del libre mercado de transporte público, y estas fallas imponían límites de factibilidad a la libre competencia y por ello se hacía necesaria la re-regulación.

Sin embargo, existe una brecha entre los conceptos de congestión y sobreoferta en Estache et al. (2004): si bien congestión y contaminación eran fallas del libre mercado del transporte público, la meta intermedia que proponía era reducir la sobreoferta, mediante una nueva organización industrial de empresas medianas que puedan invertir en buses más grandes y que trabajen en eficiencia productiva. Al suponer que al eliminar la sobreoferta y al agrandar los buses se reducía la congestión, se había encontrado la solución. Así, Estache presenta un modelo que optimiza el servicio mediante un número reducido de buses, frecuencia, o valor del pasaje, sin mostrar la variable "congestión". Así también, Ardila (2008) ya no trata sobre la congestión, sino más bien, sobre la sobreoferta como el problema a ser solucionado.

\section{La variable de sobreoferta}

La sobreoferta mide la "ocupación de vehículo" o el "llenado de pasajeros de vehículos" mediante un porcentaje cuyo valor es $100 \%$ en el año base. Influye sobre la rentabilidad del transporte público, la calidad del servicio, y la eficiencia productiva. En Lima y Callao se utilizó la sobreoferta base 1990, hasta bien entrado 2005, creyéndose que 1990 sería un año normal para el transporte público porque era la observación más cercana anterior al decreto legislativo 651 de julio de 1991 — que permitió la libre entrada de operadores al mercado del transporte público con tarifas desreguladas. No obstante, 1990 era el segundo ańo consecutivo de una caída considerable de la oferta de vehículos de transporte público, tanto que la idea de regresar al pasado de 1990 habría sido irrealista porque con toda seguridad iba a llevar a una crisis de baja oferta del transporte público. Cuando iban a implementarse los sistemas de buses rápidos promovidos por el Banco Mundial se dijo que se debía erradicar la sobreoferta del mercado. Sin embargo, no hubo acuerdo sobre una cifra exacta de la sobreoferta; en 1999 se estimó en el Proyecto Vitrina que la sobreoferta era de 1583 vehículos sobre la ruta del proyecto, mientras que en 2002 el Proyecto de Sistema de Buses Rápidos estimaba la sobreoferta en 30500 vehículos con relación a 1990 o $50 \%$ de la oferta, pero que solamente 10481 vehículos iban a ser erradicados porque sus rutas se superponían al proyecto, y finalmente se identificaron 3500 vehículos que iban a ir al chatarreo con fondos donados por agencias internacionales (Martinez 2008). Es decir, no hubo una cifra exacta de sobreoferta.

También hay otras estimaciones de sobreoferta. En el caso de Lima y Callao se ha recomendado el retiro de los buses que sean antiguos, o demasiado pequeńos como combis y/o coasters, con el fin de reducir la congestión y la contaminación (Tapia et al., 1998). Públicamente, las autoridades han expresado por un breve período, su deseo de retirar vehículos de transporte público desde 15000 vehículos de tamaño variado hasta 5000 buses estándar de $12 \mathrm{~m}$, refiriéndose a los Corredores Complementarios o Corredores Azules que se están implementando desde $2014^{5}$. A raíz de la variedad de

5 Elcomercio.pe, 29 enero de 2014. 
cifras de sobreoferta, esta variable no ha sido utilizada pues la estimación de la sobreoferta, sea por conteos, o por modelos de oferta y demanda de transporte público, es una tarea más allá del alcance de este artículo científico ${ }^{6}$. La sobreoferta no es necesariamente una externalidad. Es posible entender un aumento de oferta de buses que produzca una mejora en la calidad con un servicio más frecuente y con más asientos disponibles. Este artículo científico se ocupa más bien, de la externalidad de la congestión, ya que ha sido bien claro en decir que la externalidad es la congestión y que la medida a aplicarse es la reducción del número de vehículos del transporte público.

\section{Sistemas de buses rápidos}

Por otro lado los sistemas de buses rápidos ${ }^{7}$ utilizan carriles segregados para el uso exclusivo de buses y para protegerlos de la congestión, tal como recomendado por el Banco Mundial desde 1975. Se sabe por Gardner et al. (1991) que estos carriles segregados llamados busway alcanzan altos niveles de capacidad y de velocidad. Este artículo también calcula el efecto congestión de segregar tales carriles.

Cuando los carriles segregados para buses operan en libre mercado y sin organización operativa centralizada, llegan a congestionarse tanto entre los mismos buses que su velocidad comercial es limitada (Ardila et al., 2000). En esos casos y otros que sea conveniente, los sistemas de buses rápidos ${ }^{8}$ incrementan la velocidad de estos carriles segregados que bajan los accidentes, mediante operaciones centralmente coordinadas de buses grandes articulados, recolección automática de pasajes, y accesos especiales para los pasajeros (Diaz et al., 2000). Este nuevo sistema fue considerado tan eficiente que se ha recomendado extender sus funciones a la de una institución planificadora de todo el transporte de buses (Hook, 2005). Estos conceptos han sido aplicados en Lima como el "Metropolitano" y "Pro-Transporte".

\section{Legislación de vías saturadas}

También existe una legislación peruana que establece que si las vías de transporte se encuentran saturadas, se autoriza a las autoridades municipales a intervenir el transporte público de buses para garantizar el uso eficiente de las vías ${ }^{9}$. En ocasión de la implementación de los corredores complementarios o Corredores Azules, la autoridad municipal

\footnotetext{
6 También está más allá del alcance de este artículo la discusión entre formales e informales o autorizados e ilegales, pues que para el año 2001 todos los vehículos cumplían la legislación vigente basada en el libre mercado.

7 Carriles de buses = carriles segregados para el uso exclusivo de buses de transporte público; en inglés busway o bus-lane, luego llevados al grado de sistemas de buses rápidos.

8 Sistemas de buses rápidos = son Carriles de buses con nivel elevado operativo y tecnológico (en inglés, BRT o Bus Rapid Transit).

9 Ley 27181 Ley General de Transporte y Tránsito Terrestre 1999, permite que las Municipalidades Provinciales declaren vías saturadas, sea por congestión o contaminación, con fines de implementar concesiones de rutas de transporte publico para corregir estas externalidades.
} 
ha declarado vías saturadas a todo el conjunto de vías donde hay transporte público ${ }^{10}$, medida legal que se aplicaría a razón que se implementen los corredores mencionados. Así, al implementarse el Corredor Azul de las avenidas Tacna-Arequipa, se reemplazó 101 combis, 2076 cústers y 191 buses antiguos que también servían otras vías, con una flota de 150 buses de tamaño estándar de $12 \mathrm{~m}^{11}$. Esta relación equivalía a reducir 750 vehículos/hora de capacidad promedio de 33 pasajeros hasta 150 buses/hora de capacidad de 80 pasajeros, reduciendo la capacidad del transporte público en $-52 \%$ en los segmentos más transitadas.

Sin embargo, la definición de "saturadas" no es igual que "congestionada" o "atasco", toda vez que una vía puede estar saturada al $100 \%$ de su capacidad con un grado de saturación $\mathrm{v} / \mathrm{c}=1^{12}$, y sin embargo, operar al $60 \%$ de su velocidad de flujo libre, de acuerdo con cálculos sobre datos de (Autoridad Autónoma del Tren Eléctrico, 2002), muy por encima de una velocidad mínima de "atasco" de $6 \mathrm{~km} / \mathrm{h}$. Por ello, se recomienda que las vías saturadas sean estudiadas cuidadosamente en sus volúmenes vehiculares, en sus velocidades, y en sus tiempos de retraso por vehículo. Por lo tanto, al diagnosticar la saturación con el coeficiente $\mathrm{v} / \mathrm{c}=1$ es posible que se incurra en sobreestimación. Por ello, este artículo puede dar más datos al respecto.

\section{Metodología de ASIGNACIÓN DINÁMICA DE TRÁFICO}

La metodología consiste en aplicar un modelo de asignación dinámica de tráfico para calcular la influencia de los flujos de buses sobre la congestión. Primero, se procede a estimar la velocidad promedio de la red de transporte. La congestión se mide como la pérdida de velocidad causada por los flujos de buses, como la reducción de la velocidad entre una red sin flujos de buses y otra red con flujos de buses. Con tal fin, se debe aislar este fenómeno sin permitir ninguna otra influencia en la estimación. Para ello es que, se supone que la capacidad de transporte no varía, que no se implementan proyectos de transporte público adicionales, y que se realiza un mantenimiento de las vías tal que su capacidad no se reduzca. También se supone que la variación de los flujos de buses no afecta a la demanda de transporte público, porque podría ser que el número de buses se reduzca y que la frecuencia baje, pero que los buses sean más grandes y limpios de tal manera que logre un servicio similar al anterior tal que la demanda de transporte público de buses no tuviera por qué disminuir. Es decir, no se incluyen efectos de sustitución en el transporte, pues el fenómeno a estudiar es bastante específico y es la influencia del número de buses sobre la congestión, y por ello es que no se simulan cambios de

\footnotetext{
10 Acuerdo de Concejo 194 de Municipalidad Metropolitana de Lima de 2014 que declara vías saturadas a toda la red vial de transporte público por presentar niveles de congestión y de contaminación que comprometen la calidad de vida de sus habitantes y para ello se convocan licitaciones para corregir estas externalidades del mercado mediante la definición de un número limitado de agentes operadores.

11 Elcomercio,pe de 26 de julio 2014 y días siguientes.

$12 \mathrm{v} / \mathrm{c}=$ volumen / capacidad.
} 
demanda entre los modos de transporte público o privado. De incluirse el efecto sustitución, el fenómeno a estudiarse no queda aislado y la discusión lleva al examen de un sinnúmero de otras variables tales como la ocupación de los buses, número de asientos, precio de los autos. Es más, reducir el número de buses para reducir la congestión supone una sobreoferta preexistente en su literatura (ver más arriba en página 49). Por ello es que, al suponer en este artículo la demanda fija sin sustituciones, se supone también la existencia de sobreoferta en forma consistente con la literatura ${ }^{13}$.

Los modelos de planificación de transporte vienen equipados con cuatro modelos secuenciales, llamado en conjunto modelo de 4-etapas: producción de viajes, distribución geográfica de viajes, partición modal de los viajes (entre buses y autos o taxis), y asignación de tráfico. Este artículo utiliza la asignación de tráfico porque tiene como insumo la capacidad de vías (que es la oferta), la matriz origen-destino (que es la demanda) y da como resultado la velocidad de la red de transporte.

Los modelos de asignación de tráfico consisten en un algoritmo que se inicia al calcular el árbol de caminos de costos mínimos entre orígenes y destinos de la red de transporte, como una secuencia de nodos (intersecciones) y/o enlaces (calles o pistas) tomando en cuenta la longitud de cada calle, su capacidad, y la cantidad de vehículos usando esa calle. Las calles tienen una capacidad a partir de la cual la velocidad va bajando a medida que se llena de autos, por virtud de una curva función volumendemora, cuyas ecuaciones se presentan más abajo en la Tabla 1.

La causalidad teórica de los modelos de asignación de tráfico es la siguiente: cada automovilista busca reducir su costo de viaje en ítems de tiempo, costo de combustible, peajes, y mantenimiento. Cada pasajero de transporte público también minimiza sus costos de viaje en ítems de tiempos de caminata, precio del pasaje, tiempo de viaje en vehículo, y esperas en el paradero. Todo se reúne en un modelo de programación no-lineal que asigna los flujos de tráfico, minimizando los costos individuales de los automovilistas y pasajeros, con las restricciones de que las calles utilizadas en forma secuencial, una después de otra, forman caminos origen-destino. Se espera que los flujos y los tiempos sean positivos. Luego, se calculan otra vez los caminos mínimos y se inicia otra iteración, hasta alcanzar un mínimo de cambios respecto del valor anterior, donde el algoritmo se detiene cuando una solución aproxime un nivel pequeño de error ${ }^{14}$. Un modelo de asignación de tráfico puede estimar los cambios de velocidad causados por los flujos de buses.

\footnotetext{
13 Se utilizan los efectos sustitución en proyectos que atraen usuarios de autos y taxis al transporte público, pero en este caso estudiado sería absurdo esperar una transferencia a causa de la reducción de la capacidad productiva del transporte público pues tendría un efecto opuesto. En el caso del Metropolitano, se sustituyó un busway de 13000 pasajeros por hora por dirección en la Vía Expresa por otro de 25000 y allí si procede el análisis de sustitución.

${ }^{14}$ La literatura sobre modelos de asignación de tráfico tiene varias décadas de desarrollo y forman la base de una rama especial llamada Transportation Science. Ya que se usa solamente un software, no se presenta literatura al respecto.
} 
El software Dynasmart asigna la demanda de tráfico vehicular en períodos de cada cinco minutos, calculando medidas de rendimiento de la red de transporte. Combina modelos de asignación de tráfico, con las funciones densidad-velocidad de cada vía, y con los algoritmos de ruta más corta, la que se reactualiza cada cinco minutos desde la ubicación del vehículo en tránsito, todo en una sola estructura de simulación visual y asignación de tráfico (US-DOT, 1994 y 2007). Este software cumple con modelar reducciones de capacidad de las vías y por ello puede ser utilizado para evaluar los efectos de los flujos de buses que reducen la capacidad de vías y/o evaluar los efectos de la segregación de carriles para uso exclusivo de buses.

Previamente se calculan los flujos de buses por tramo. El EMME3 tiene un control específico para calcular los flujos de buses en cada enlace a partir de la frecuencia, ruta, número de vehículos y tipo de vehículo utilizado en la línea de buses en unidades de carros de pasajeros (UCP). Este control supone que el número de líneas de buses es de menos de 200, por lo que se tuvo que correr varias veces, también supone que los buses hacen todas las operaciones dentro de la hora, resultándose en un promedio de la hora típica, así la ruta de buses dure más de una hora. En resumen, con los softwares Dynasmart y EMME3 se producen las estimaciones de la variable velocidad dados los flujos de buses.

\section{CONSTRUCCión DE La BASE DE DATOS}

\section{Detalles sobre la matriz origen-destino del Plan Maestro}

El Plan Maestro de la referencia Ministerio de Transportes y Comunicaciones 2005 establece que la matriz origen-destino se construye sobre la encuesta de viajes por hogares. Esta encuesta se basa en un muestreo por área geográfica de 35000 hogares censales, el $2 \%$ del total, distribuidos adecuadamente en Lima y Callao, muestra que es considerada estándar y suficiente. La muestra está estratificada en cuatro sectores enumerando desde el de mayores al de menores ingresos: AB (19,1\%), C (24\%), D (39,5\%), y E (17,4\%). Los resultados son luego expandidos a toda la población, por área geográfica, obteniéndose la matriz origen-destino. La matriz origen-destino es complementada con datos de encuestas de taxis y de camiones, en especial sobre los viajes en vacío. Esta matriz es calibrada en dos etapas: i) se compara con el valor real de los conteos de la línea cortina, que llegan a 3,25 millones de personas diarias, correspondiendo un ajuste de la matriz hacia arriba por factores entre 1,0 a 2,6 dependiendo del modo de transporte utilizado, y ii) se compara la asignación de tráfico en software Jica-Strada que da flujos por segmentos de vías, con el valor real de conteos sobre esos mismos segmentos, hasta alcanzar un ajuste de $\mathrm{R}^{2}=0.86$.

Otro método de calibración aplicado en otra parte, ajusta la capacidad de vías del modelo, o de funciones volumen-demora, dada una matriz origen-destino preexistente, mediante una regresión entre resultados del modelo de asignación de tráfico de tramos 
de la red con los de esos mismos tramos, hasta lograr una alta correlación $\mathrm{R}^{2}$, lo cual se recomienda para ciudades en desarrollo donde no se conocen los valores de capacidad o de velocidad de flujo libre por lo que se les puede ajustar a discreción. En el caso de este artículo científico se aplica una tercera clase de calibración, esta vez sobre las funciones volumen-demora que son estimadas a partir de regresiones sobre los datos de campo disponibles, los cuales incluyen capacidad y velocidad de flujo libre, con el objetivo de reproducir los efectos de la congestión por tramos de vía, véase la Tabla 1.

Sobre la encuesta de viajes de hogares se estiman los 32 modelos de regresión lineal sobre generación y atracción de viajes por estrato y por propósito de viaje, cuyo ajuste obtiene indicadores $R^{2}$ desde 0,85 a 0,98 . Estas ecuaciones incluyen la propiedad del automóvil, el ingreso económico y su probabilidad de usar auto o taxi. Luego, se estiman 25 modelos no lineales de distribución de viajes por estrato y por propósito de viaje cuyos coeficientes de ajuste $R^{2}$ varían entre 0,47 a 0,82 . Finalmente, se aplican los 10 modelos de partición modal, cuyos parámetros son estimados en una muestra sobre 2400 personas que son considerados suficientes para un buen ajuste estadístico. Todas estas ecuaciones se van a aplicar luego, para obtener la matriz origen-destino 2025, sus pasos previos se describen a continuación.

De acuerdo al Plan Maestro, para obtener la matriz origen-destino 2025 se ubica el crecimiento poblacional de $1.5 \%$ anual de manera geográfica de acuerdo a los planes de la ciudad. Igual el crecimiento del producto bruto regional doméstico que se estimó en 4,3\% anual. Los estratos $\mathrm{AB}$ y $\mathrm{C}$ crecerán, el $\mathrm{D}$ decrecerá, y el $\mathrm{E}$ quedará igual. Finalmente, se aplican las ecuaciones arriba enumeradas sobre los datos proyectados de la población en 2025 obteniéndose la matriz origen-destino 2025 .

\section{Detalles sobre las base de datos utilizadas para este artículo}

Primero, se reproduce la red de transporte sobre la base del Plan Maestro Ministerio de Transportes y Comunicaciones (2005), mostrada en la Figura 1. La red se compone de 446 zonas de tráfico ${ }^{15}, 6542$ nodos $^{16}$, y 14977 enlaces $^{17}$. El área geográfica alcanza $90 \mathrm{~km}$ sur-norte por $40 \mathrm{~km}$ este-oeste. La más poblada es el área entre el puerto del Callao, el Centro de Lima, y San Isidro-Miraflores, que es intensiva tanto en autos y taxis como también en buses. Las vías se han verificado visualmente por medio del Google Earth dando como resultado una red un poco diferente de la red del Plan Maestro, sobre todo en el número disponible de carriles por vía y en detectar vías sin pavimentar o en proyecto.

\footnotetext{
15 Zona de tráfico es una zona censal suficientemente pequeña para conformar una unidad de origen o destino de viajes de transporte urbano.

16 Nodo es como una intersección pero no todas las intersecciones son modeladas.

${ }^{17}$ Enlaces son tramos de calles que puede tomar cualquier longitud pero que pueden incluir varias intersecciones semaforizadas o sin semáforos en cada tramo.
} 
Figura 1. Red de transporte de Lima y Callao

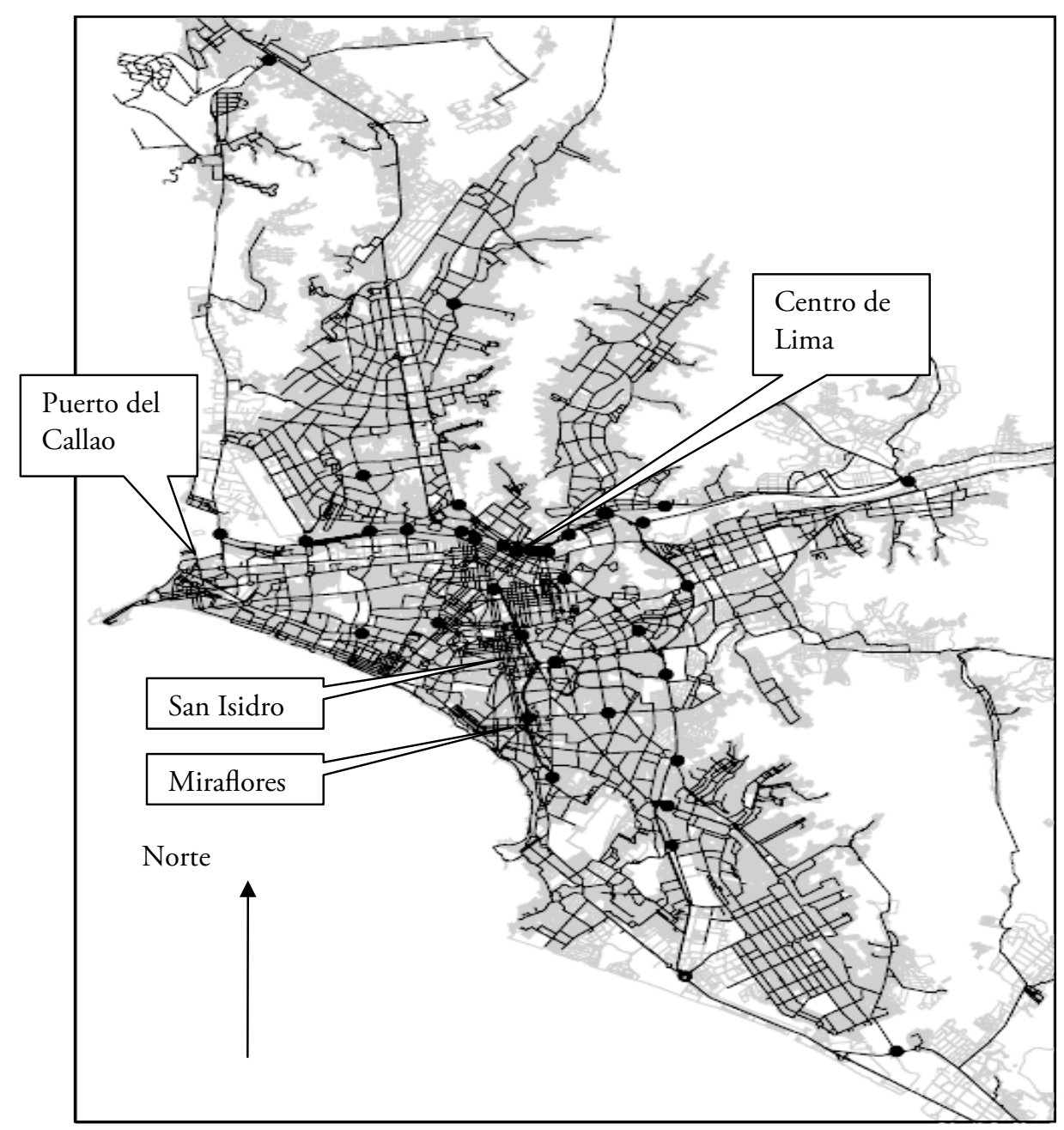

Segundo, se calculan los flujos de buses sobre cada enlace usando el software EMME2 para 2005 con los datos de frecuencia por ruta, y flota autorizada, de la Municipalidad. Para 2025 los buses crecen por un factor de 1,34 (Ministerio de Transportes y Comunicaciones, 2005). Se consideran aquellos flujos de buses en tráfico mixto con autos y taxis. Los flujos de buses llegan a $5500 \mathrm{UCP} / \mathrm{h}^{18}$ en plaza Bolognesi con seis carriles, a $1800 \mathrm{UCP} / \mathrm{h}$ en plaza Dos de Mayo con siete carriles, o a $1500 \mathrm{UCP} / \mathrm{h}$ en prolongación Tacna-Rímac con tres carriles por sentido.

${ }^{18} \mathrm{UCP} / \mathrm{h}=$ unidades de carros de pasajeros por hora, que es por dirección y por sentido. Las equivalencias de flujo vehicular son de 3,00 UCP por bus, 2,25 por cúster, y 1,25 por combi y 1,00 por auto o taxi, y 3,00 por camión de carga. 
Tercero, se adaptan los modelos de flujos de tráfico a Dynasmart pues se detectan diferencias entre los resultados esperados y los obtenidos por el software, similares a penalidades por intersecciones no señalizadas. Al no tener datos de campo sobre capacidad de descarga de cada intersección ni de sus giros, se ha optado por realizar un ajuste adicional de la velocidad de flujo libre y la capacidad de vía. Se describen estos modelos como: $S=S f\left(1-\frac{D}{D j}\right)^{\alpha}$, donde $S$ es velocidad, D es densidad ${ }^{19}$, Sf es velocidad de flujo libre, Dj es densidad de "atasco" a velocidad mínima de $4 \mathrm{mph}$, y alfa es un factor a calibrarse; y $F=S * D$, donde $F$ es flujo vehicular. Para calibrar la diferencia, se usa una red de 4 millas de largo (más del doble de la distancia mínima con que se ha medido la velocidad en campo) con intersecciones sin señalizar y similares niveles de tráfico. Se muestran los resultados calibrados en la Tabla 1.

Tabla 1. Modelos de flujo de tráfico ${ }^{21}$

\begin{tabular}{ccccccc}
\hline & \multicolumn{3}{c}{ Modelo calibrado } & \multicolumn{4}{c}{ Valores de campo } \\
\cline { 2 - 7 } Tipo de vía & $\begin{array}{c}\text { velocidad autos } \\
\text { mph }\end{array}$ & $\begin{array}{c}\text { capacidad } \\
\text { UCP/h / carril }\end{array}$ & $\begin{array}{c}\text { Alfa } \\
\text { velocidad autos } \\
\text { (veloc. buses }{ }^{20} \text { ) } \\
\text { mph }\end{array}$ & $\begin{array}{c}\text { capacidad } \\
\text { UCP/h / carril }\end{array}$ & $\mathbf{R}^{2}$, F $\left(^{*}\right)$ \\
\hline Arterial D & 35 & 1850 & 1,27 & $23(15)$ & 850 & $0,29,12$ \\
Arterial E & 40 & 1800 & 1,50 & $25(22)$ & 860 & $0,37,--$ \\
Colectora & 30 & 1700 & 1,37 & $24(23)$ & 600 & $0,24,6$ \\
Arterial F & 35 & 1350 & 1,38 & $22(20)$ & 900 & $0,64,--$ \\
Arterial B & 30 & 1200 & 1,88 & $18(12)$ & 500 & $0,87,--$ \\
Carretera (una) & 60 & 2200 & 2,75 & $38(29)$ & 1350 & $0,37,5$ \\
Expresa (una) & 75 & 2400 & 1,25 & 62 & 1600 & $0,76,68$ \\
\hline
\end{tabular}

$(*)$ los “- - " son valores del estadístico F que son perdidos en la estimación del momento.

Arterial D.- avenidas de tres o más carriles por sentido, de tráfico mixto, con intersecciones a nivel, y semaforizadas.

Arterial E.- avenidas de dos carriles por sentido de tráfico mixto, con intersecciones a nivel, y semaforizadas.

Colectora.- avenidas de un carril por sentido que conectan las calles locales a las avenidas; el tránsito es controlado en las principales intersecciones usando señales y marcas en el piso.

Arterial F.- avenidas de un carril por sentido, de tráfico mixto, con intersecciones a nivel y semaforizadas.

Arterial B.- vías segregadas de dos carriles especiales para transporte público al lado de dos carriles exclusivos para transporte privado de autos y taxis, por sentido; con intersecciones a nivel, y semaforizadas.

Carretera.- vías de gran capacidad de tres o más carriles por sentido, de tráfico mixto, con accesos controlados a nivel, e intersecciones muy distanciadas, con paraderos de buses y peaje de vehículos.

Expresa.- vías de gran capacidad de tres o más carriles por sentido, de tráfico privado exclusivo para autos y taxis, con accesos y salidas por rampas, sin interferencias transversales.

19 Densidad es el número de vehículos por kilómetro por carril, presentes en una avenida en un determinado momento.

${ }^{20}$ Velocidad comercial de buses, incluye subidas y bajadas de pasajeros en los paraderos.

${ }^{21}$ Vía Expresa Javier Prado todavía tiene menos de 5 millas y tiene una curva que le baja la velocidad a menos del estándar de una Vía Expresa, es decir que, sin ampliarse ni mejorarse, pues se debe considerar una sola Expresa la del Paseo de la República, aunque se asigna su función volumen-demora a Expresa. 
Se tomaron los datos de velocidades y de volúmenes de manera simultánea, a bordo de autos-de-prueba, (Autoridad Autónoma del Tren Eléctrico, 2002) sobre 36 tramos de vías cuya distribución geográfica replica el tamaño de muestra necesaria para calibrar el modelo del proyecto del tren eléctrico de 1998, son tramos de más de una milla de extensión (distancia mínima para tomar datos de velocidades-segmento), los tramos incluyen intersecciones semaforizadas y no-semaforizadas, con varias observaciones para cada tramo tanto en hora pico como en flujo libre. Las velocidades de buses se tomaron a bordo de los buses, simultáneamente y en similares condiciones y número de observaciones que las descritas para autos y taxis. En adelante, la velocidad de buses es una proporción fija entre 0,64 y 0,95 de la velocidad de los demás vehículos, coincidiendo las proporciones más altas con las vías menos congestionadas. Los procedimientos seguidos para calcular la velocidad de flujo libre y la capacidad por carril, son estándares generalmente aceptados para estos fines. Las estimaciones originales de las funciones volumen-demora tienen un $\mathrm{R}^{2}$ de 0,51 de promedio, los $\mathrm{R}^{2}$ más bajos corresponden a segmentos con poca congestión.

Cuarto, se calcularon las matrices de demanda origen-destino OD por hora desde la matriz OD de 24 horas del Plan Maestro de Ministerio de Transportes y Comunicaciones (2005). Para ello, se utilizaron varios factores del propio Plan Maestro tales como grado de ocupación de pasajeros por auto $(2,00)$ y por taxis $(0,71)$ de los conteos de tráfico de la referencia Ministerio de Transportes y Comunicaciones (2005), porcentaje de viajes realizado por autos y taxis por cada motivo (trabajo, estudio y otros), número de viajes motorizados por motivo por hora, medios de transporte por motivo de viaje, y número de viajes generados por 14 áreas geográficas. Puede haber una discrepancia entre la matriz de la hora pico y la matriz de la hora pico publicada por Ministerio de Transportes y Comunicaciones (2005), basada sobre el pico de los flujos sobre la línea cortina del río Rímac, la que podría estar muy ligeramente subestimada al constatarse que sus puentes soportan un tráfico vehicular muy alto formando meseta por dos horas seguidas, aunque los conteos de pasajeros sí forman un pico.

Quinto, se simularon variaciones de demanda por períodos de cinco minutos, cuyos datos se recolectaron mediante conteos dentro del contrato 194-2005-CONCYTEC/OAJ, estos datos se tomaron con mayor detalle que un conteo normal y por cada cinco minutos, en vez de quince que es el período técnicamente estándar. Se realizaron estos conteos sobre la ruta del tren eléctrico, desde Puente Atocongo hasta Hospital Dos de Mayo, y sus fines fueron cuantificar la variabilidad del flujo de tráfico en cuatro direcciones, y presentarlos como funciones distribución estadística de variabilidad de tráfico en tendencias creciente, decreciente, y meseta, necesarias para modelos dinámicos. En el caso de los camiones de carga, los viajes se distribuyeron usando los orígenes y los destinos especializados en productos tales como combustibles con refinería, productos agrícolas con mercados, y según las horas permitidas para dicho tráfico. Finalmente, se tomó un valor del tiempo de los usuarios de autos y taxis igual a 2,6 soles por hora (Martínez y Cornejo, 2003). 


\section{Plan de estimación de CONGestión POR Flujos de buSes}

Se corre el software Dynasmart para los ańos 2005 y 2025, considerando cuatro casos: "teórico", “+buses", "max_buses", y "carril_buses". El caso "teórico" tiene solo flujos de autos, taxis, y camiones. El caso "+buses" reduce la capacidad de las vías en la cantidad exacta de los flujos de buses que pasan por allí, permitiendo calcular una cantidad precisa de congestión; esto se origina en un procedimiento estándar de asignación de tráfico que considera a los flujos de buses como fijos y se usan para reducir la capacidad de uso del tráfico privado, pero este artículo aprovecha este cálculo intermedio que usualmente no se muestra al lector para calcular la congestión causada por los buses. El caso "max_buses" reduce el número de carriles cuando los flujos de buses alcanzan $50 \%$ o más de la capacidad de un carril, calculando un máximo posible de congestión por flujos de buses; este es un procedimiento no-estándar usado en países en desarrollo, por el cual, en vez de reducirle la capacidad a la vía por los flujos de buses, se le reduce su número de carriles, da como resultado una posible sobreestimación del efecto de los flujos de buses, aunque facilita el cálculo del modelo; como resultado se obtiene que el 15\% de la red de transporte sufre la reducción de carriles en 2005 y el 22\% en 2025 (usualmente no se muestran estos datos al lector). El caso "carril_buses" segrega un carril por dirección sobre 24 kms de una ruta Este-Oeste sobre segmentos compuestos por diversos tipos de vía, tomando como ejemplo un proyecto publicado en Municipalidad Metropolitana de Lima-Pro-Transporte (2011); este caso sirve para estimar el punto de discusión muy frecuente de si segregar un carril de una vía para un sistema de buses rápidos va a causar congestión.

Las comparaciones se realizan a tres niveles: Primero, se comparan los valores de los casos de "+buses" y "max_buses" sobre el caso "teórico" lo que da como resultado la congestión promedio y la máxima, respectivamente causada por el transporte público de buses sobre la red de transporte. Segundo, se compara el año de 2025 sobre el año de 2005 con el fin de obtener la congestión causada por el incremento de viajes en autos, taxis, y camiones de carga. Tercero, se compara el caso "carril_buses" sobre el caso “+buses" con el fin de obtener la congestión adicional causada por segregar un carril sobre $24 \mathrm{kms}$ de vía.

Los escenarios permiten el análisis de la congestión causado por los flujos de buses para compararlos con otros factores de interés, tales como infraestructura, cambios en el tamaño de los autos y otros. Hay casos que necesitan escenarios, como los planes maestros o los proyectos de inversión pública. Sin embargo, en este artículo es factible proceder sin incluir tales escenarios para ser estrictos con alcanzar el objetivo propuesto.

\section{MEDIDAS DE RENDIMIENTO}

Las medidas de rendimiento son las siguientes: velocidad en millas por hora (mph), valor del tiempo de viaje en dólares \$time, costos monetarios de vehículos en dólares $\$$ cost, y tiempo de viaje entre pares de origen y destino ODtt en minutos. Estas medidas se refieren a los viajes de vehículos de autos, taxis, y camiones de carga mientras que los 
flujos de buses van a tener un rol pasivo de sí o no están presentes, con una velocidad de buses que no es necesario calcular por ser proporcional a la velocidad de autos y taxis en factores fijos de entre 0,65 a $0,95^{22}$.

La velocidad es un resultado del software Dynasmart ponderado por el número de viajes de vehículos por cada hora.

El valor del tiempo de viaje \$time se calcula multiplicando las matrices $10^{*} 1500$ por minuto de la densidad por minuto por enlace, por la matriz de longitud del enlace, por las proporciones de ocupación vehicular y por el valor del tiempo por hora.

Los costos monetarios \$cost, incluyen peajes, mantenimiento de vehículos, y costo de combustible. El costo de mantenimiento de los vehículos se calcula multiplicando los vehículo-horas por el costo de mantenimiento por hora: 3 centavos de dólar por auto, 4,4 centavos por camión, y 4,2 por taxi (Ministerio de Transportes y Comunicaciones, $2005)^{23}$. El costo de combustible se obtiene multiplicando la matriz de velocidad por enlace por minuto por el consumo de combustible por kilómetro, dado por una escala de 8 niveles de velocidad, y por el número de vehículos presente en cada enlace en ese minuto.

El tiempo de viaje promedio entre orígenes y destinos ODtt es el promedio simple de la matriz de los tiempos de viaje de todos los pares origen-destino.

\section{RESULTADOS DEL ANÁLISIS DE LA CONGESTIÓN VEHICULAR}

La Figura 2 presenta los estados de la velocidad y de la demanda para cada una de las horas del día, utilizando una red de transporte que ya tiene incluidos los flujos de buses en sus calles; las horas-pico de la demanda ocurren a las siguientes horas: 7 a.m. ( $11 \%$ de la demanda), 8 a.m. (7\%), 1 p.m. (8\%), y 6 p.m. (7\%). En 2005, la máxima velocidad alcanza $42 \mathrm{mph}$ a las 2 a.m. y la mínima a $10 \mathrm{mph}$ a las 7 a.m.; mientras que en 2025, la máxima alcanza $40 \mathrm{mph}$ y la mínima $6 \mathrm{mph}$. En 2005 se modelan 2,3 millones de viajes de vehículos de autos, taxis, y camiones de carga (cifra suma de las matrices correspondientes de 24 horas 2005 del Plan Maestro Ministerio de Transportes y Comunicaciones $2005)^{24}$, cuyo máximo ocurre en hora-pico con 260000 viajes y 35000 buses-horas. En 2025 se modelan 4,2 millones de viajes de vehículos (matrices de 24 horas 2025 de Ministerio de Transportes y Comunicaciones 2005), cuyo máximo en hora-pico es de 450000 viajes y 40000 buses-horas. Entre 2005 y 2025, la demanda de la hora-pico aumenta en $+73 \%$ de viajes de vehículos particulares y la de los de transporte público en $+14 \%$ de buses-hora, mientras que la velocidad de hora-pico se reduce en $-40 \%$.

\footnotetext{
22 Hay proyectos de transporte público de buses que incrementan su velocidad; pero, en tráfico mixto con autos y taxis, la velocidad de buses siempre van a ser una proporción de la velocidad de autos y taxis.

23 Es posible que estos valores parezcan demasiado bajos, pero resultan de encuestas en Lima y Callao, y puede ser que el mantenimiento sea económico por costumbres locales o por un clima benigno.

${ }_{24}$ Son cifras fijas del Plan Maestro sobre las cuales no corresponde un análisis de sensibilidad en este artículo, en otro estudio especial se podría hacer un análisis de sensibilidad porque para ello se necesitaría replicar todo el proceso de elaboración de la matriz OD 2025.
} 
Nótese que durante el mismo día, y sin variar los flujos de buses, los flujos de autos, taxis, y camiones reducen la velocidad de la red de transporte en -76\% en 2005 (comparando 2 a.m. con 7 a.m.), y que la reducción de la velocidad empeora a $-85 \%$ en 2025 , mientras que las reducciones causadas por el transporte público son bastante menores como se verá en la Tabla 2.

La Tabla 2 presenta las medidas de rendimiento. El total de los flujos de buses reducen la velocidad de la red entre $-12 \%$ en 2005 y $-16 \%$ en 2025, con topes máximo de $-20 \%$ en 2005 y $-25 \%$ en 2025 . En contraste, el crecimiento de los viajes de autos, taxis y camiones reduce esta velocidad entre $-37 \%$ en 2005 y $-41 \%$ en 2025 . Esto significa que el crecimiento de viajes de autos, taxis, y camiones origina más del doble de congestión que todos los flujos de buses. Si se considerara la meta de reducir la mitad de los buses, se incrementaría la velocidad en $+7,0 \%{ }^{25}$. Si se considerara las metas de 2014 de reducir entre $10000^{26}$ y $15000^{27}$ de buses, se incrementaría la velocidad entre $+2,8 \%$ y $4,2 \%$ (extrapolando linealmente los resultados de velocidad de Tabla 2), y, si se consideraran buses de mayor tamaño la velocidad aumentaría solo entre 2 y $2,8 \%$. Esto significa, considerar que la reducción de buses como política contra la congestión es mucho menos efectiva de lo que se creía para bajar la congestión, y que se debiera administrar mejor el transporte por autos, taxis y camiones.

Figura 2. Estados de la velocidad y de la demanda por hora del día

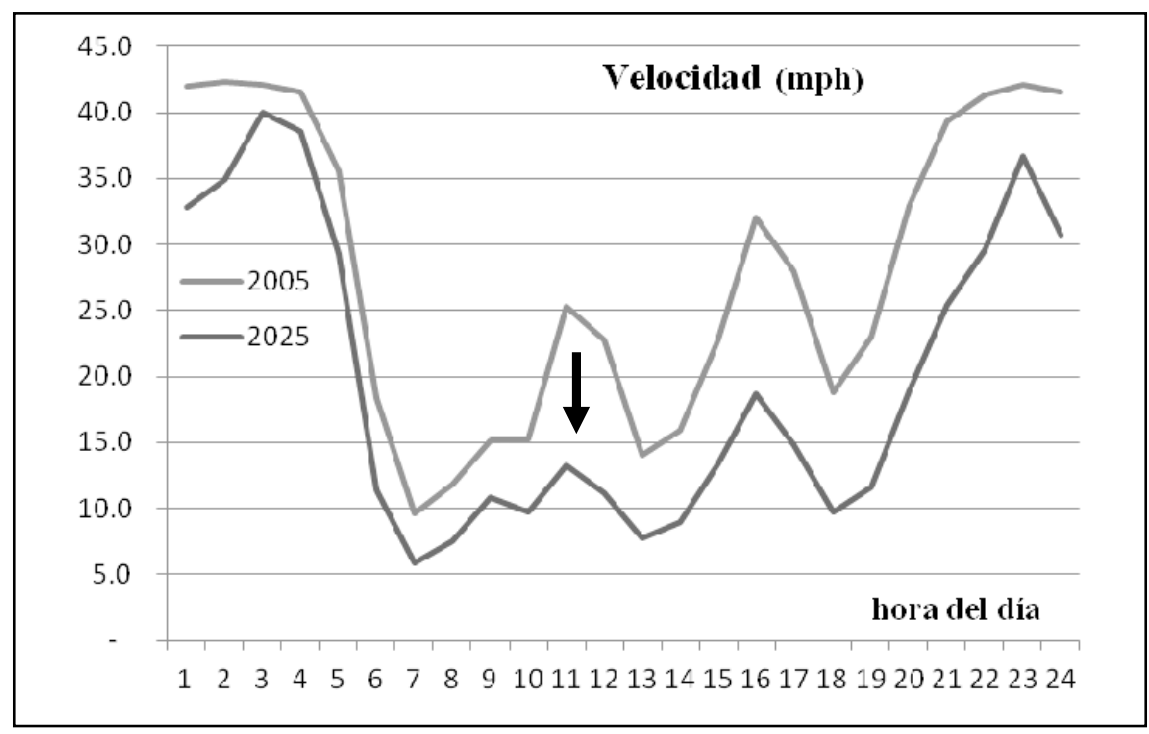

$257 \%=(12 \%+16 \%) /(2($ promedio del período $) * 2(\operatorname{mitad}$ de la flota $))$.

26 Elcomercio.pe del 29 enero 2014.

27 www.youtube.com video "Conoce los avances del Sistema Integrado del Transporte de Lima”. Municipalidad de Lima, publicado el 24 de octubre de 2013. 


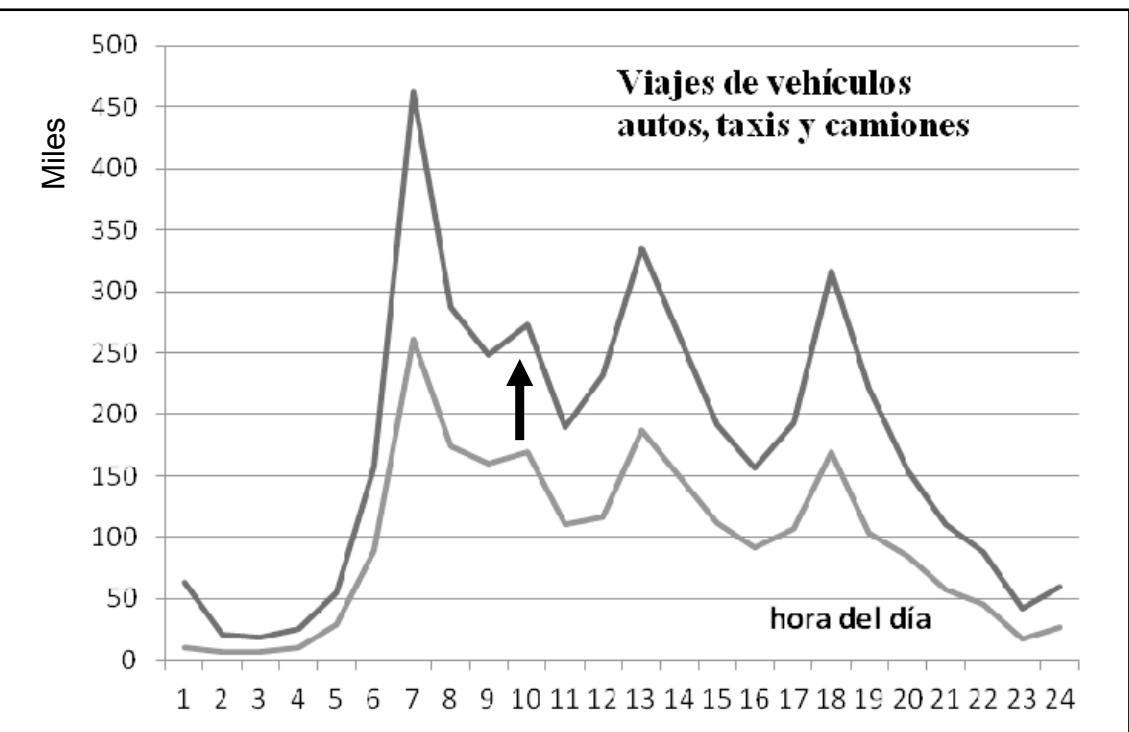

Según la Tabla 2, los flujos de buses incrementan los costos de tiempo (\$time) sobre los usuarios de autos, taxis y camiones entre $+9 \%$ en 2005 y $+11 \%$ en 2025 , con topes máximos entre $+11 \%$ en 2005 y $+12 \%$ en 2025 . Sin embargo, el crecimiento de los viajes de autos, taxis y camiones entre 2005 a 2025, incrementan costos entre $+131 \%$ a $+136 \%$. Reducir la mitad de los buses con vehículos más grandes, permitiría ahorrar $+3,3 \%$ de costos de tiempo de viaje, cuarenta veces menos que el aumento de los costos causados por el crecimiento de transporte usando autos, taxis y camiones.

En la Tabla 2 se presentan los costos monetarios (\$cost) de peajes, mantenimiento, y combustible. Los flujos de buses elevan muy poco los costos monetarios, entre $+1 \%$ y $+4 \%$ entre 2005 y 2025, no muy diferente de cero. El tráfico desplazado por el transporte público de una avenida, se muda a otro camino sobre la cual el diferencial de costos es pequeño. La nueva ruta puede demorar más tiempo o ser más larga, pero si tiene mayor velocidad, los costos de combustible pueden ser menores. Así, los costos monetarios de congestión quedan amortiguados porque hay múltiples caminos. Por el otro lado, si la reducción de buses del transporte público liberara espacio en determinada avenida, una cantidad de autos y taxis escogería esa avenida liberada porque la ve más veloz que antes, y seguirían entrando más autos y taxis a esa misma avenida hasta un nivel tal, que esta avenida llegue a estar tan "llena" como antes de la reducción de buses, es decir que, regresaría a un nivel de congestión similar al de antes, con una velocidad similar o poquito mayor que antes. El efecto total de reducir el número de buses del transporte público es de $+2,5 \%$, es muy pequeño comparado con el efecto del crecimiento de viajes de autos, taxis, y camiones sobre el período 2005-2025 el cual llega a $+55 \%$. 
Tabla 2. Medidas de congestión por casos de transporte público de buses $(*)(* *)$

\begin{tabular}{|c|c|c|c|c|}
\hline $\begin{array}{c}\text { año/ } \\
\text { variable }\end{array}$ & $\begin{array}{c}\text { teórico } \\
\text { (1) }\end{array}$ & $\begin{array}{c}+ \text { buses } \\
(2) \\
\%=(2) /(1)-(1)\end{array}$ & $\begin{array}{c}\underset{(3)}{\max \_b u s e s} \\
\%=(3) /(1)-(1)\end{array}$ & $\begin{array}{c}\text { carril_buses } \\
(4) \\
\%=(4) /(1)-(1)\end{array}$ \\
\hline \multicolumn{5}{|c|}{2005} \\
\hline $\begin{array}{l}\text { velocidad } \\
\text { (mph) }\end{array}$ & 20,9 & $\begin{array}{c}18,3 \\
-12 \%\end{array}$ & $\begin{array}{c}16,8 \\
-20 \%\end{array}$ & $\begin{array}{l}18,0 \\
-2 \%\end{array}$ \\
\hline $\begin{array}{l}\text { \$time } \\
(000.0)\end{array}$ & 3235 & $\begin{array}{l}3524 \\
+9 \%\end{array}$ & $\begin{array}{c}3585 \\
+11 \%\end{array}$ & $\begin{array}{l}3577 \\
+2 \%\end{array}$ \\
\hline $\begin{array}{l}\text { \$cost } \\
(000.0)\end{array}$ & 5464 & $\begin{array}{l}5517 \\
+1 \%\end{array}$ & $\begin{array}{l}5255 \\
-4 \%\end{array}$ & $\begin{array}{l}5515 \\
+0 \%\end{array}$ \\
\hline $\begin{array}{c}\text { ODtt } \\
\text { (minutos) }\end{array}$ & 12,6 & $\begin{array}{r}13,3 \\
+6 \%\end{array}$ & $\begin{array}{l}13,1 \\
+3 \%\end{array}$ & $\begin{array}{l}13,3 \\
+0 \%\end{array}$ \\
\hline \multicolumn{5}{|c|}{2025} \\
\hline $\begin{array}{c}\text { velocidad } \\
(\mathrm{mph})\end{array}$ & $\begin{array}{l}13,2 \\
-37 \% \\
\end{array}$ & $\begin{array}{c}11,1 \\
-16 \% \\
-39 \% \\
\end{array}$ & $\begin{array}{c}9,9 \\
-25 \% \\
-41 \% \\
\end{array}$ & $\begin{array}{l}10,9 \\
-2 \% \\
-39 \%\end{array}$ \\
\hline $\begin{array}{l}\text { \$time } \\
(000.0)\end{array}$ & $\begin{array}{r}7475 \\
+131 \% \\
\end{array}$ & $\begin{array}{c}8306 \\
+11 \% \\
+136 \% \\
\end{array}$ & $\begin{array}{c}8385 \\
+12 \% \\
+134 \% \\
\end{array}$ & $\begin{array}{c}8364 \\
+1 \% \\
+134 \% \\
\end{array}$ \\
\hline $\begin{array}{l}\$ \text { cost } \\
(000.0)\end{array}$ & $\begin{array}{r}8492 \\
+55 \%\end{array}$ & $\begin{array}{l}8801 \\
+4 \% \\
+60 \%\end{array}$ & $\begin{array}{c}8034 \\
-5 \% \\
+53 \%\end{array}$ & $\begin{array}{l}8814 \\
+0 \% \\
+60 \%\end{array}$ \\
\hline $\begin{array}{c}\text { ODtt } \\
\text { (minutos) }\end{array}$ & $\begin{array}{r}14,8 \\
+17 \%\end{array}$ & $\begin{array}{c}16,1 \\
+9 \% \\
+21 \%\end{array}$ & $\begin{array}{c}15,4 \\
+4 \% \\
+18 \%\end{array}$ & $\begin{array}{c}16,2 \\
+1 \% \\
+22 \%\end{array}$ \\
\hline
\end{tabular}

(*) Porcentajes en itálicas corresponden al cambio porcentual de todas las medidas de rendimiento entre 2005 y 2025 , causada por el incremento de viajes de autos, taxis y camiones.

(**) Resultados corresponden a los efectos sufridos por los flujos de autos, taxis, y camiones de carga causados por los flujos de buses, siendo la velocidad de los buses proporcional a la velocidad de los demás vehículos.

La Tabla 2 también presenta el tiempo de viaje ODtt. Nótese el bajo valor promedio de entre 13 y 15 minutos que corresponde largamente a viajes cortos de autos y taxis dentro del triángulo Callao-Lima-Miraflores y alrededores ${ }^{28}$. Los flujos de buses aumentan este tiempo de viaje en $+7,5 \%$ en promedio. Pero, esta influencia del transporte público de buses es pequeña comparada con el incremento del tiempo de viaje de $+19 \%$ en promedio causada por el incremento de viajes por autos, taxis y camiones en el período estudiado.

28 Falta incluir los tiempos de caminata, búsqueda y maniobras de estacionamiento, y pago del mismo. 
Finalmente, en la cuarta columna de datos se aprecia que segregar carriles de buses sobre $24 \mathrm{~km}$ sobre una ruta este-oeste reduce la velocidad en tan solo - $2 \%$. Las demás variables muestran tendencias similares. Por ello, se recomienda segregar carriles exclusivos para sistemas de buses rápidos donde sea adecuado.

Figura 3. Reducción de la velocidad causada por el transporte público a lo largo del día (porcentaje de la velocidad)

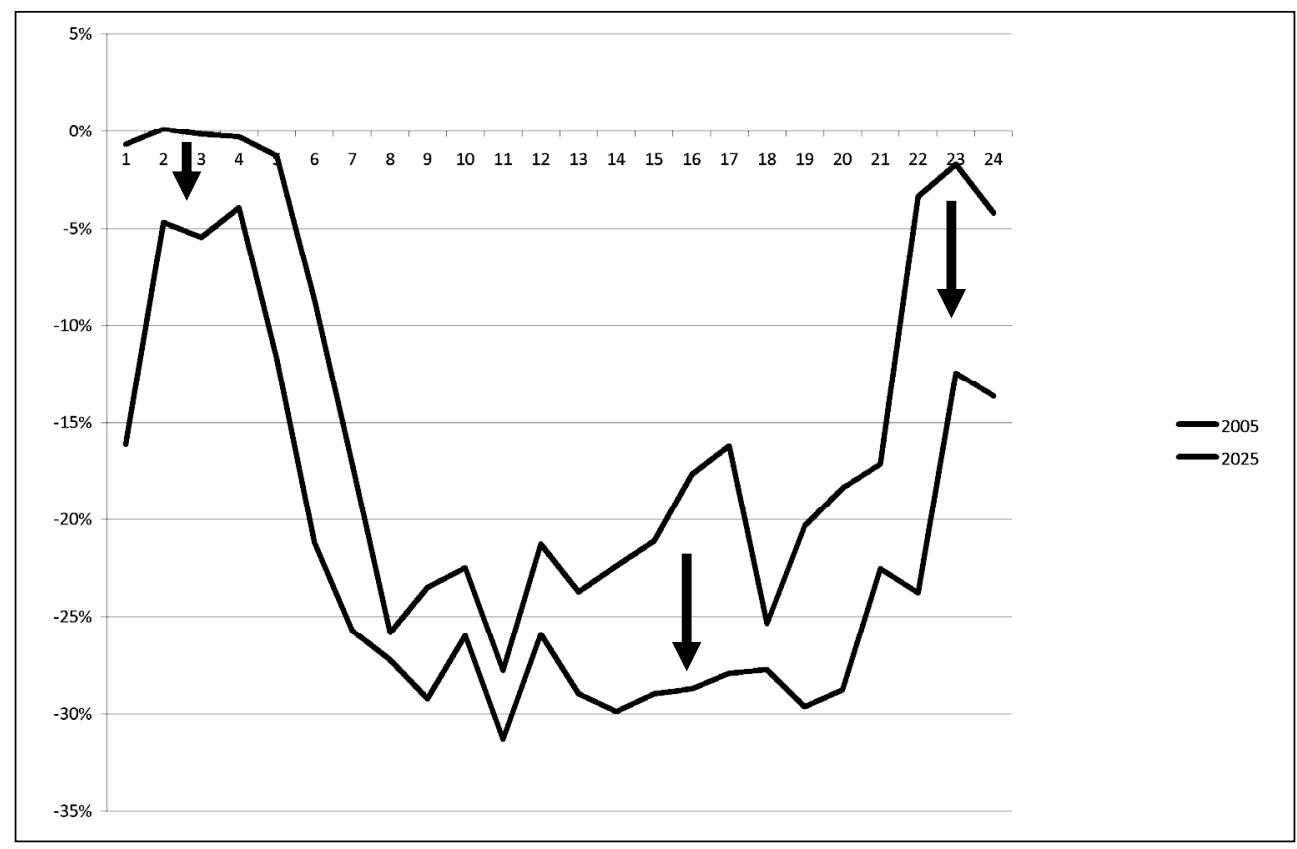

La Figura 3 presenta la reducción de la velocidad a lo largo del día causado por los flujos de buses del caso "max_buses" 29 . La curva de 2005 muestra que el transporte público rebaja la velocidad de la red en $-25 \%$ durante tres horas separadas del día (8 a.m., 11 a.m. y 6 p.m.), mientras que la curva de 2025 muestra que rebaja la velocidad entre $-25 \%$ y $-30 \%$ durante catorce horas entre 7 a.m. a 8 p.m., pero con un pico a las 11 a.m. De reducirse los flujos de buses a la mitad, se obtiene una mejora del $+11 \%$ de velocidad en promedio durante las horas desde 7 a.m. a 6 p.m., con un máximo de mejora de $+14 \%$ a las 11 a.m. En 2014, las autoridades municipales fijaron la meta de reducir entre 10000 y 15000 buses, y siendo los nuevos buses de mayor tamańo, se incrementará la velocidad de la red entre $+4.4 \%$ y $+6.6 \%$ durante las horas del día y entre $+5,6 \%$ y $+8,4 \%$ durante la hora-pico más gravemente congestionada por los flujos de buses.

29 “max_buses” influencia máxima posible del flujo de buses, 160\% de “+buses” influencia promedio. 
La Figura 4 presenta la reducción de la velocidad causada por el transporte público sobre un segmento de vía. El caso presentado es el de "carril_buses" que segrega un carril para el sistema de buses rápidos. Se nota que los momentos de gran congestión generados por el flujo de buses en 2005 corresponden a períodos de algunos minutos durante el día y durante las horas pico. También se aprecia que en 2025, estos períodos se alargan hasta por 25 minutos, períodos en los cuales se congestionan mucho las vías por los flujos de buses. Se concluye que la reducción de la velocidad causada por los flujos de buses dura cierta cantidad de minutos durante el día y que, en el resto del día, la reducción de la velocidad causada por la influencia del transporte público es generalmente menor a $20 \%$ y casi siempre menor a $60 \%$, mientras que los momentos graves de $95 \%$ de reducción de la causada por el transporte público prevalecen solamente en períodos de menos de 25 minutos. En la Figura 4 aparecen también valores negativos que significan que los flujos de buses causan retrasos a los demás vehículos en sus trayectorias hacia el segmento analizado, sobre todo en momentos previos a las horas-pico.

La Figura 5 presenta la influencia del flujo de buses sobre la congestión por zonas o segmentos de vías seleccionadas. Los gráficos corresponden al caso “+buses” en el año base 2005. En la parte (a) se presenta la Av. Javier Prado-La Marina sobre tres segmentos de este a oeste. En el primer segmento por Av. La Molina, se nota por contados minutos la influencia de los flujos de buses sobre la congestión, la que está asociada con una falta de infraestructura adecuada en los accesos a La Molina. El hecho que la influencia sea mayor en la dirección Este indica que autos y taxis regresan en una distribución más esparcida. En el segundo segmento a la altura de Av. Arenales la influencia por contados minutos es más frecuente, en las horas pico, lo que corresponde al acceso al centro de San Isidro. En el tercer segmento por la Av. La Marina, no hay esa influencia ni en contados minutos pues corresponde a una zona menos céntrica y con menos necesidad de infraestructura.

En la parte (b) se presenta la Vía Expresa del Paseo de la República sobre cuatro segmentos de Norte a Sur, donde no se permiten flujos de buses pero que sufren la influencia del flujo de buses sobre otras vías de la red de transporte. En el primer segmento a la altura del Estadio Nacional, se nota la influencia por contados minutos de los flujos de buses sobre la congestión, en una situación de cercanía al centro de Lima. En el segundo segmento a la altura de Av. Canadá, se nota que esta influencia se exacerba aunando dos condiciones, la dirección al centro de Lima y la falta de infraestructura ya que en este segmento confluyen los autos y taxis que vienen del sur y los que vienen de San Borja y de Surco, vía la Av. Javier Prado. En el tercer segmento a la altura de Av. Córpac, la influencia vuelve a ser, por contados minutos y durante las horas pico, correspondiente al acceso al centro de San Isidro. En el cuarto segmento a la altura de Av. Benavides, la influencia es muy poca o puede considerarse inexistente. 
Figura 4. Reducciones de la velocidad en segmentos de una avenida (porcentaje de la velocidad)

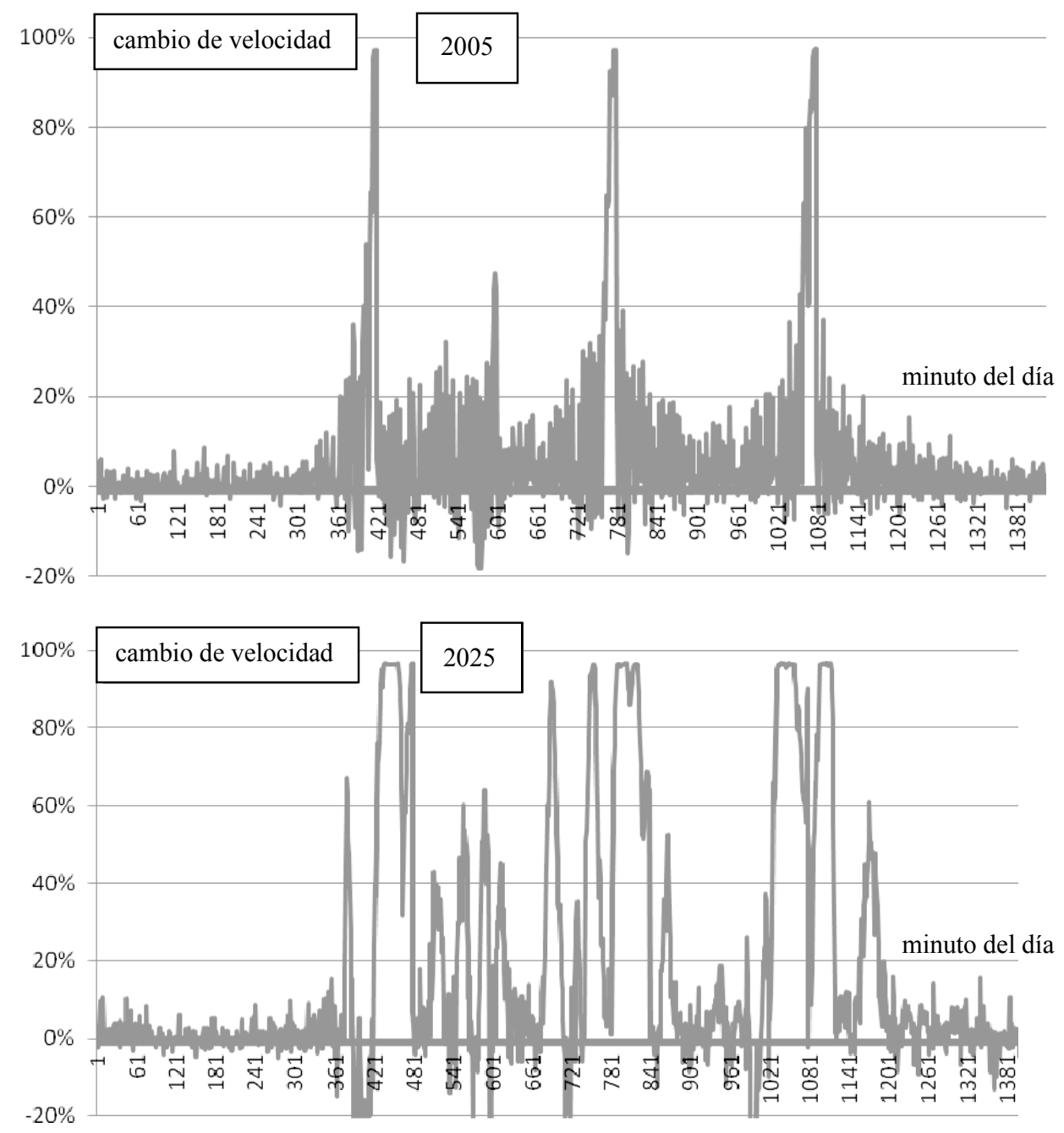

De las observaciones de la Figura 5 se puede decir que, los flujos de buses influyen sobre la congestión de las avenidas por contados minutos durante las horas pico, que esta influencia se manifiesta más en zonas cercanas a centros de la ciudad y en zonas de poca infraestructura relativa a la demanda, y finalmente, que hay zonas donde la influencia es prácticamente inexistente.

En consecuencia, la legislación de vías saturadas debiera aplicarse más durante las horas-pico, y en las zonas céntricas, o que sean escasas de infraestructura de transporte. 
Figura 5. Influencia del flujo de buses sobre la congestión por zonas o segmentos en avenidas seleccionadas (porcentaje de la velocidad)

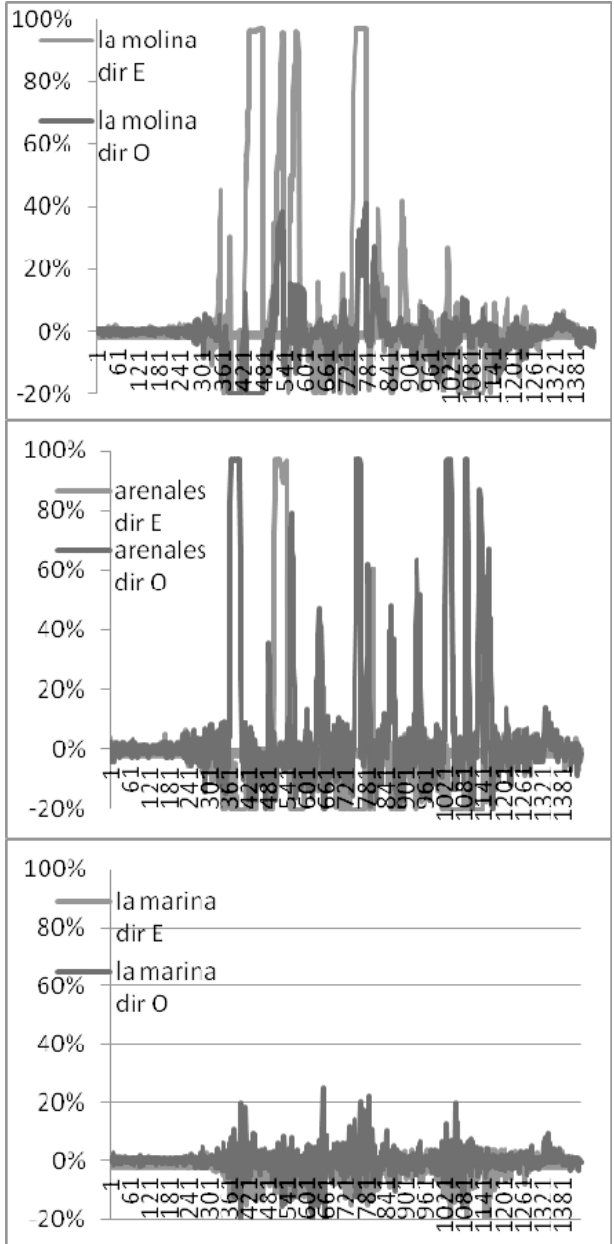

a) Corredor Av. Javier Prado-Av. La Marina de este a oeste.

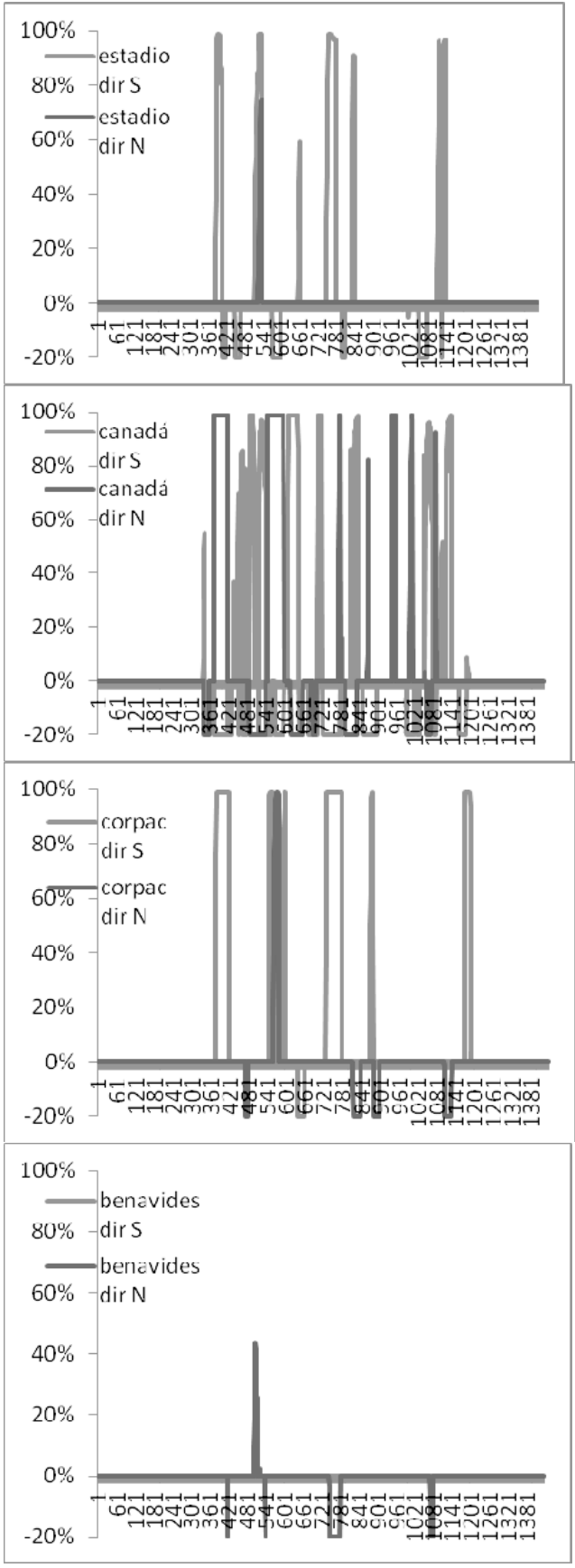

b) Vía Expresa Paseo de la República de sur a norte. 


\section{CONCLUSIONES Y RECOMENDACIONES SOBRE EL ANÁLISIS DE LA CONGESTIÓN VEHICULAR}

Del análisis de la congestión, se ha encontrado que en promedio por día, la influencia de los flujos de buses sobre la velocidad es de $14 \%$, que en el promedio por hora, esta influencia es de cerca del $25 \%$ durante las horas pico de las 8 a.m., 11 a.m. y 6 pm, y que cuando se mide por minuto, esta influencia llega al $90 \%$ aunque por contados minutos durante las horas pico, y que está localizada en zonas céntricas y/o zonas de escasa infraestructura vial con relación a la demanda de transporte. De reducirse el 50\% del número de buses, la velocidad se incrementaría en $7 \%$ en la red de transporte, de $7 \%$ a $11 \%$ durante ciertas horas, y de $9 \%$ a $14 \%$ a las 11 a.m., la hora pico de influencia de los buses. De lograrse las metas de 2014 de reducir entre 10000 y 15000 vehículos de transporte público y de recomponer la flota con buses grandes estándares, entonces la velocidad se incrementaría entre 2,0\% y 2,8\%. Estos resultados son desbordados por completo, cuando se comparan con la caída de la velocidad de $-39 \%$ causada por el simple crecimiento de los flujos de autos, taxis y camiones entre 2005 y 2025. Es decir, actuar sobre los flujos de buses resulta muy inefectivo para combatir la congestión.

Por ello, se recomienda desestimar la medida de reducir el número de buses para combatir la congestión. Más bien, se recomienda el uso por horas de carriles contrasentido, por el cual los carriles de la dirección no-pico pasen a servir a la dirección pico durante esas horas, y también identificar las soluciones de construcción de una mejor infraestructura, de semaforización y otras soluciones que se puedan planificar. Sería también recomendable estudiar políticas de reducción de la congestión que actúen sobre los flujos de autos, taxis y camiones, en vez de sobre los buses.

También se ha estimado la influencia de segregar un carril de vía para buses rápidos siendo su influencia de $-2 \%$ sobre la velocidad, una influencia muy pequeña que invita a instalar de hasta 3 a 7 de esos buses rápidos en las rutas donde se considere apropiado, segregando un carril de vía por distancias de entre 20 a $30 \mathrm{~km}$ sin que haya una pérdida significativa de la velocidad de la red de transporte del área metropolitana.

Finalmente, la legislación de vías saturadas para intervenir el transporte público debiera enfocarse sobre las horas-pico, así como dedicarse a las zonas céntricas o zonas de poca infraestructura.

\section{EL TRANSPORTE PÚBLICO DE BUSES Y LA CONTAMINACIÓN AMBIENTAL}

\section{El PROBlema DE LA CONTAMINACión AMBIENTAL Y LAS ALTERNATIVAS DE SOLUCióN}

El transporte de las ciudades en desarrollo, genera tal contaminación ambiental que ha motivado que el Banco Mundial recomiende desde 1996, que las decisiones gubernamentales sobre transporte deban ser ambientalmente sostenibles y dirigidas a reducir tanto la contaminación del aire como también el calentamiento global. Para ello, se deben 
calcular los costos de salud provocados por la contaminación e incluir las alternativas de combustible y de tecnología vehicular disponibles (Gwilliam, 2002).

En las ciudades en desarrollo, los niveles de concentración de material particulado (pm10) son varias veces más elevados que en las ciudades industrializadas por el mayor uso del combustible diésel (Gwilliam et al., 2004). Además de ello, la rápida motorización ${ }^{30}$ empeora los problemas de contaminación por las elevadas emisiones de los autos particulares (Kokaz et al., 2002).

Las políticas más importantes para reducir la contaminación ambiental del transporte urbano son las siguientes, las mismas que suelen ser complementarias entre sí:

Convertir a combustibles más limpios es una solución frecuente para los problemas de calidad del aire. El gas natural ha sido adoptado en Bangkok, Teherán, y Santiago (Heil et al., 1998). Sin embargo, para que esta política sea efectiva se necesita de un gasoducto desde el propio yacimiento para que el precio de gas natural sea competitivo con el diésel tradicional y lograr una conversión eficiente (The World Bank, 2001). En Lima y Callao, se han implementado las mezclas de etanol y biodiésel, y se vende a precios bajos gas natural vehicular y gas líquido de petróleo. Actualmente se está realizando la inversión pública en los proyectos de la Refinería de Talara en Piura-Perú, para producir diésel sin sulfuros; y en el Gasoducto del Sur desde Cusco-Perú, para obtener un abastecimiento suficiente de gas natural a bajo costo; la inversión total de ambos proyectos es de 6,3 billones ${ }^{31}$ de dólares. Su ventaja es reducir directamente la contaminación (Gobierno Peruano, 2004). Se han prohibido gasolina con plomo desde 2004 y diésel con azufre desde 2011.

El etanol ha sido introducido en Lima y Callao desde 2011, como una mezcla de $7,8 \%$ con gasolina donde reemplaza complementos oxigenadores reduciendo la contaminación; el etanol anhidro se produce de la caña de azúcar del norte del Perú. Biodiésel también ha sido introducido desde 2011, como una mezcla de 5\% con diésel; el biodiésel se produce en 35\% de la palma aceitera en la Amazonía del Perú y el resto se importa del extranjero (Bustamante, 2013).

El gas natural vehicular iba a originar un boom comercial luego de que el primer gasoducto llegara a Lima y Callao (Gobierno peruano, 2004). El sistema de buses rápidos "Metropolitano" basó sus planes de negocios en un gas natural vehicular de bajo precio. Según cifras publicadas, los autos y taxis que usan gas natural vehicular o gas licuado de petróleo llegan a cerca de $10 \%$ para cada combustible.

Inspección y mantenimiento consiste en programas de revisión técnica de vehículos para controlarles las emisiones de contaminantes mediante límites estándares. Ha sido recomendada como una política costo-efectiva porque la flota vehicular de ciudades

\footnotetext{
30 Se llama motorización al proceso de compras masivas de autos particulares con fines de transporte urbano, en contraste con el tradicional uso intensivo de buses o trenes en ciudades en desarrollo.

31 Billones $=$ mil millones .
} 
en desarrollo es antigua, y pueden corregirse las emisiones con facilidad (Shalizi et al., 1994). Puede bajar el consumo de combustible de -3\% a -7\% reduciendo las emisiones de $\mathrm{CO}^{22}$ (Armann et al., 2005). Complementa la alternativa de cambios a combustibles más limpios porque mantiene la eficiencia de motores luego de la conversión (Kojima et al., 2001). En Lima y Callao, esta alternativa ha sido promocionada para controlar la aplicación de estándares de emisión de contaminantes (Gobierno Peruano, 2001, 2004) y para complementar la alternativa de reducir el número de buses al mantener las emisiones de los buses en ciertos límites o retirarlos del mercado (Gobierno peruano, 2004 y Defensoría del Pueblo, 2008).

Reducir la flota de buses del transporte público, muy frecuentemente recomendada para Lima y Callao, a imitación de Santiago (Malbrán, 1998) ${ }^{33}$. En Thomson et al. (2001) se explica que, al re-regular el transporte público se reduce el número de buses, y se alivian la congestión y la contaminación. Más recientemente, en (Defensoría del Pueblo, 2008), se recomienda no solamente reducir el número de buses, pero también reemplazarlos por buses más grandes que utilicen gas natural vehicular, y que las empresas pequeñas sean reemplazadas por empresas medianas.

Sin embargo, esta alternativa ha encontrando graves dificultades inesperadas que han retrasado su implementación por más de 15 años sin llegar a la meta propuesta. Estas dificultades son las siguientes: i) acciones judiciales de amparo que protegen el derecho al trabajo de los transportistas ${ }^{34}$ (Quispe, 2007), ii) diferencias entre las Municipalidades de Lima, Callao y Huarochirí sobre el número adecuado de vehículos de transporte público a ser autorizado ${ }^{35}$, iii) retrasos en promulgar una legislación adecuada para retirar vehículos antiguos ${ }^{36}, \mathrm{y}$, iv) descontento de los pasajeros por un nuevo sistema con menos buses ${ }^{37}$.

$32 \mathrm{CO}_{2}=$ contaminante dióxido de carbono, causante del calentamiento global, y de cambios del clima global.

33 La re-regulación en Santiago de Chile empezó justamente con una sentencia judicial sobre contaminación ambiental alrededor de una avenida sufrida por los vecinos por el elevado flujo de buses, por lo cual se inicia la subasta pública de rutas de transporte público por esa avenida.

34 (www2.caretas.pe/2001/1653/lima.phtml) del 18 enero 2001.

35 Hasta en fechas recientes, como en (www.andina.com.pe del 24 abril 2014), donde el Municipio de Lima dice que desiste temporalmente de trabajar en el Régimen de Gestión Común entre Lima y el Callao, y que los buses autorizados por el Callao no tendrán concesión de 10 años en Lima aunque podrán participar de las licitaciones de los Corredores a licitarse.

36 En 2017, la Municipalidad Provincial del Callao ha aprobado un cronograma de retiro de unidades del transporte público por antigüedad de manufactura, con fechas de salida de operaciones que van desde los manufacturados hasta 1991 en 2017 y los manufacturados en 2007 en 2019.

37 Los pasajeros del Corredor Azul de Av. Tacna-Arequipa declararon que hay número insuficiente de buses, que hay largas colas de espera en los paraderos, que tienen que realizar extensas caminatas al paradero y que los buses operan demasiado llenos (elcomercio.pe del 10 septiembre 2014 y días cercanos). 
Además, el Estado Peruano no ha presupuestado los costos sociales de cerca de 90000 desempleados causados por la erradicación de los 30000 buses (Martínez 2008), pues hay un ingreso perdido de familias pobres por 346 millones de dólares anuales (calculado de Martinez, 2009). Estas consecuencias actúan contra las funciones económicas gubernamentales de mantener el nivel de empleo y de favorecer una distribución más equitativa del ingreso.

\section{RAZONES Y PARTICULARIDADES DE LA IMPLEMENTACIÓN DE COMBUSTIBLES MÁS LIMPIOS EN CIUDADES EN DESARROLLO}

Los combustibles más limpios sustituyen a la gasolina y al diésel para reducir emisiones de contaminantes, diversificar fuentes de energía y reducir las emisiones de $\mathrm{CO}_{2}$ desde el ciclo de producción del combustible. Los combustibles más limpios son: etanol, biodiésel, gas natural vehicular y gas licuado de petróleo.

El etanol y el biodiésel tienen altos costos de producción y por ello requieren de subsidios (Gwilliam et al., 2004). Se les prefiere porque la caña de azúcar o el frijol soya consumen $\mathrm{CO}_{2}$ durante su crecimiento. El etanol tiene un impacto similar al biodiésel que reduce el $\mathrm{CO}_{2}$ por más de $75 \%$. Cuando los precios de los combustibles suben mucho, el etanol y el biodiésel son más competitivos y dan oportunidades para que los países en desarrollo mejoren su calidad de aire (Armann et al., 2005).

Los combustibles a gas son también utilizados para reducir las emisiones de $\mathrm{CO}_{2}$. El gas natural vehicular emite entre $-15 \%$ y $-20 \%$ menos de $\mathrm{CO}_{2}$ comparado con la gasolina. El gas licuado de petróleo tiene similares características pero su abastecimiento es generalmente más limitado (Armann et al., 2005). Una particularidad crucial de los combustibles a gas es la necesidad de un gasoducto desde el yacimiento hasta la ciudad, que provea un abastecimiento suficiente a bajo costo, que lo haga muy ventajoso frente al diésel y así convertir los vehículos a combustibles de gas voluntaria y masivamente. Si no se implementa el gasoducto, resulta demasiado costoso distribuir el gas a causa de su infraestructura de reabastecimiento (Gwilliam et al.; 2004, Kojima et al., 2001; Kojima 2001).

Por último, se ha recomendado reemplazar los buses a diésel por buses a gas natural vehicular, basándose sobre la reducción de emisiones que ahorran costos de salud. Sin embargo, los buses a gas natural vehicular son más caros en precios de compra, costos de mantenimiento, y costos de operación. Además, necesitan de una infraestructura bastante cara. Entonces, los operadores de transporte público necesitan de un precio muy bajo del gas natural con el fin de compensar por estos costos más altos, y también necesitan de una regulación férrea de proteccionismo de sus operaciones comerciales para que puedan garantizar su factibilidad financiera. Esta es una razón adicional para construir el número apropiado de gasoductos que permita un precio bajo del gas natural (Kojima, 2001). 


\section{MODELO DE ASIGNACIÓN DINÁMICA DE TRÁFICO Y PROCEDIMIENTOS COMPLEMENTARIOS}

Los modelos de transporte son necesarios en las ciudades en desarrollo para simular las emisiones de contaminantes y optimizar su transporte, sobre todo en el contexto de un importante transporte público de buses y de una rápida motorización (Rogers et al., 2001).

Originalmente, los modelos de una sola carretera usaban factores de emisiones, vehículos-milla, y velocidad promedio para calcular las emisiones (JHK\&A, 1978). Los modelos de una red de transporte, necesitan de los flujos vehiculares para calcular las emisiones al multiplicar por los factores de emisión, de acuerdo a la velocidad de cada enlace de la red (USDOT 1979). El modelo MOBILE calcula los factores de emisiones por tipo de vehículo y por tipo de carretera, en gramos por vehículo-milla de contaminantes (Chatterjee et al., 1997; Dowling et al., 2005). Hay modelos que calculan emisiones por viaje $\mathrm{OD}$ vehicular, por tipo de vehículo, por motivo de viaje, y por hora del día; sea por "enlace" o por "par" origen-destino (Bai et al., 2007).

En Lima y Callao, la relación velocidad-factores_de_emisiones se obtiene de (Municipalidad Metropolitana de Lima, 2005) lo cual empieza por un análisis de combustibles del Ministerio de Transportes y Comunicaciones AB, Haningen, Sweden en octubre 2002 (financiado por el contrato 7117971 del Banco Mundial para la Iniciativa de aire Limpio de Lima y Callao). Luego, se utilizaron estadísticas de tecnología vehicular, antigüedad, mantenimiento, y combustible, y se obtuvieron los factores de emisión usando el software COPERT, la versión europea del MOBILE (Municipalidad Metropolitana de Lima, 2005). Estas curvas se han utilizado en estudios para el Sistema de buses rápidos en Lima y Callao en 2005 y en Arequipa en 2010.

A partir del modelo Dynasmart se obtiene la matriz de velocidad por enlace por minuto, la cual se baja a una hoja Excel, donde se obtiene la cantidad de kilómetros por minuto que se multiplican por el factor de consumo de combustible de la curva velocidad-combustible de (Municipalidad Metropolitana de Lima, 2005). Luego se calculan estos galones por minuto multiplicando por el número de vehículos presentes en cada enlace por cada minuto, los cuales se agregan para totalizar el combustible consumido por hora. De manera similar se calculan las emisiones de los contaminantes pm10, sox, nox, $\mathrm{y} \mathrm{pb}^{38}$.

Otro enfoque es el de la demanda de potencia física que utiliza un análisis paramétrico representativo para estimar emisiones segundo-a-segundo y puede producir curvas de velocidad-emisiones que incluya aceleraciones y desaceleraciones (Barth et al., 1996).

38 pm10 = material particulado en polvo de 10 micras de diámetro o menos.

sox $=$ óxidos de azufre.

nox $=$ óxidos de nitrógeno

$\mathrm{pb}=$ plomo. 
Como resultado, se ha desarrollado el software CMEM que usa perfiles de velocidad segundo-a-segundo para estimar emisiones (Barth et al., 2000).

Un estudio de las emisiones de vehículos de Lima y Callao, con datos de 2003, incluye el comportamiento de vehículos monitoreados por GPS para construir perfiles típicos de velocidad segundo-a-segundo y por rangos de velocidad y potencia; también recolectó la distribución local de tecnología vehicular (antigüedad, modelos), y estadísticas de video del uso de las vías de transporte, todo para ser usado como insumo para el software IVE (International Vehicle Emissions, similar al CMEM pero especial para ciudades en desarrollo). Estos estudios encontraron que el sistema de transporte de Lima y Callao puede emitir hasta 367 toneladas por día de nox (Davis et al., 2005, y Lents et al., 2004), un nivel de $78 \%$ de las estimaciones de este artículo científico para 2005.

En este artículo científico se presentan cuatro factores adicionales:

1) Motor frío: Se ajustan las emisiones al considerar que del $15 \%$ al $35 \%$ de los viajes vehiculares por hora empiezan con motor frío (Lents et al., 2004) por lo que deben ser multiplicados por un factor que se calcula con el software CMEM con las características climáticas de Lima y Callao, resultando factores entre 1.2 y 1.3.

2) Perfil al segundo: Se calcula el incremento de emisiones causado por aceleraciones y desaceleraciones con el software CMEM al hallar la diferencia de emisiones entre la velocidad promedio y el perfil de velocidad típico por tipo de vía: calles urbanas, carreteras y calles colectoras/distribuidoras (Lents et al., 2004); se obtienen factores de entre 1,04 a 1,56 .

3) Presencia por minuto en el segmento: Se ajusta por el número de vehículos que pasa de un enlace a otro en el período de un minuto, por lo que su presencia es contada de manera doble, este factor asciende en promedio a 0,6.

4) Costumbres de Lima y Callao: Se describe la costumbre particular de Lima y Callao de acelerar muy frecuentemente para cambiar de carriles mientras se conduce a baja velocidad, mediante un factor calculado en 1,12.

Se extrapolan las emisiones de cada contaminante al período de un año, luego se les introduce en una hoja de cálculo Excel, y se relacionan linealmente las emisiones con las concentraciones de contaminantes al promedio del año base 2005. Luego, se forma una línea proporcional en las cercanías de ese punto entre emisiones y concentraciones promedio. Este método es un aproximado al método peruano para calcular rápidamente la nueva concentración de contaminantes a partir de cambios en las emisiones de contaminantes de fuentes móviles (transporte urbano) y en proyectos de medio ambiente (CONAM, 2005; Kroger, 2005). 
Tabla 3. Cambio porcentual de la incidencia de enfermedades debido a cambios de concentración de contaminantes $\left(^{*}\right)$

\begin{tabular}{|c|c|c|c|c|c|c|c|c|c|}
\hline efecto & pm10 & $\mathrm{pm} 2.5$ & $\mathrm{SO}_{2}$ & $\mathrm{NO}_{2}$ & $\mathrm{O}_{3}$ & $\mathrm{~Pb}$ & $\begin{array}{l}\text { ratio } \\
\mathrm{x} 100 \mathrm{k}\end{array}$ & $\begin{array}{c}\text { días } \\
\text { perdidos }\end{array}$ & costo\$ \\
\hline mor1 & 1,01 & & 0,48 & & 0,59 & & 385 & & 453600 \\
\hline mor2 & 3,84 & & & & & & 385 & & 453600 \\
\hline mor3 & & & & & & 35 & 17,1 & & 453600 \\
\hline $\mathrm{ahr}$ & 1,39 & & & & 3,76 & & 217 & 7 & 1204 \\
\hline ahc & 0,98 & & & & 0,6 & & 39 & 10 & 1204 \\
\hline vse & 3,11 & & & & 3,17 & & 1081 & 1 & 206 \\
\hline dar & 7,74 & & & & & & 181383 & 1 & 12 \\
\hline $\mathrm{dpt}$ & 7,74 & & & & & & 160056 & 1 & 8 \\
\hline darm & 4,92 & & & & 2,2 & & 780000 & 0 & 6 \\
\hline asm & 7,74 & & & & 2,45 & & 10000 & 1 & 37 \\
\hline bro & 3,6 & & & & & & 707 & 7 & 176 \\
\hline $\operatorname{cin}$ & & & & & & 0,975 & 83 & 1 & 1204 \\
\hline sra & & & & 0,1 & & & 16000 & 0 & 14 \\
\hline srp & 5,62 & & 0,1 & & 1,85 & & 16000 & 1 & 14 \\
\hline bra & 11 & & & & & & 4400 & 1 & 176 \\
\hline irri & & & & 0,266 & & & 29500 & 0,5 & 25 \\
\hline lcan & & 8 & & & & & 15 & 48 & 453600 \\
\hline
\end{tabular}

$\left({ }^{*}\right)$ por $10 \mu \mathrm{g} / \mathrm{m}^{3}$, microgramos por metro cúbico en el caso de $\mathrm{Pb}$ : por $1 \mu \mathrm{g} / \mathrm{m}^{3}$

mor1 mortalidad prematura, por exposición aguda

mor2 mortalidad prematura, por exposición crónica

mor3 mortalidad prematura por 100000 adultos masculinos

ahr ingreso a hospital debido a enfermedad respiratorias

ahc ingreso a hospital debido a enfermedad cardiovascular

vse visita a la sala de emergencias

dar día de actividad restringida

dpt pérdida de días de trabajo

darm días de actividades restringidas moderadamente

asm ataques de asma

bro bronquitis crónica

cin reducción del cociente intelectual de niños

sra síntomas respiratorios en adultos

srp síntomas respiratorios en el sistema respiratorio alto, el bajo, y/o el medio

bra bronquitis aguda

irri dolor de ojos

lcan riesgo de cáncer al pulmón

pm10 Material particulado de menos de 10 micras de diámetro

pm2.5 Material particulado de menos de 2,5 micras de diámetro

$\mathrm{SO}_{2} \quad$ Dióxido de azufre

$\mathrm{NO}_{2} \quad$ Dióxido de nitrógeno

$\mathrm{O}_{3} \quad$ Ozono

$\mathrm{Pb}$ Plomo

Valores y composición de tabla de (CONAM, 2005; Kroger, 2005; Ostro, 1994; Cesar, 2002) 
Luego, los costos anuales de salud se calculan como el cambio porcentual de la incidencia porcentual de enfermedades causadas por los cambios de la concentración de contaminantes. La incidencia es el número de casos por cada 100000 habitantes, y se multiplica por el costo de un evento de la enfermedad, y luego se multiplica por el número de días perdidos por la enfermedad, si fuera aplicable (Ostro, 1994; Cesar, 2002). Esto es, simplemente la aplicación de tasas en los dos períodos de análisis.

La Tabla 3 ha sido elaborada ampliando tablas similares y tomando los valores de CONAM (2005), Kroger (2005), Ostro (1994) y Cesar (2002). Muestra el cambio porcentual de la incidencia de enfermedades debido a los cambios en la concentración de contaminantes, y también muestra el ratio de incidencia por cada 100000 habitantes, el número promedio de días perdidos en promedio por evento, así como el costo en dólares por evento. Estos datos se complementan con la relación entre emisiones y concentración de contaminantes calibrada para 2005, y se calcula el cambio del costo de enfermedades, o costos de salud, ante cambios en las emisiones de contaminantes.

Las emisiones de $\mathrm{CO}_{2}$ se calculan del total de combustible consumido por año. Los factores de emisión son $8,9 \mathrm{~kg} / \mathrm{gal}$ de gasolina, y 10,2 kg/gal de diésel. Los factores de ciclo-productivo por sobre las emisiones de combustión, ascienden a 1,47 para gasolina, 1,32 para diésel, 1,40 para E7.8, 1,27 para B5, 1,59 para gas natural vehicular, y 1,35 para gas licuado de petróleo (Wang et al., 2012; Andreoli et al., 2006; Sheehan et al., 1998; Delucchi, 2001).

\section{Plan de estimación de las alternativas de solución}

Se estiman seis alternativas para reducir la contaminación ambiental:

(1) Convertir a combustibles más limpios. Conversión desde gasolina a E7.8 sin plomo, desde diésel a B5 de bajo sulfuro, y promoción de gas natural vehicular y de gas licuado de petróleo hasta una proporción de 10\% del mercado de autos y taxis para cada combustible de gas. Caso ya implementado en Lima y Callao; calcula el efecto de la reducción de emisiones por cambiar a la nueva mezcla de 2012 desde la antigua mezcla de combustibles de $2005^{39}$.

(2) Programa de Inspección y mantenimiento. Caso implementado en Lima y Callao; calcula el efecto de este programa con una efectividad de 32\% de corrección positiva para ahorrar combustible y reducir emisiones, desde una proporción de $13 \%$ de los motores a gasolina en ineficiencia y de $22 \%$ de los a diésel. Incluye la reducción de emisiones derivadas de un mejor mantenimiento del parque vehicular al estar obligando a los propietarios de vehículos a mantenerles de manera eficiente de acuerdo a unos porcentajes de cobertura y de efectividad tal como los mencionados en este párrafo.

\footnotetext{
392012 sobre 2003 para el caso de gasolina con plomo sobre gasolina sin plomo.
} 
(3) Reducción de buses a la mitad. Caso frecuentemente recomendado para reducir contaminación a partir de la disminución de la flota del transporte público o chatarreo.

(4) Conversión de todos los taxis a gas natural vehicular. Caso hipotético que se ha dado en otras ciudades en desarrollo.

(5) Conversión de todos los buses a gas natural vehicular. Caso hipotético que merece ser analizado.

(6) Conversión de todos los buses sobre una ruta de $24 \mathrm{~km}$ a gas natural vehicular. Caso hipotético a ser analizado.

Las alternativas implementadas (1) y (2) en Lima y Callao y se comparan sus beneficios con la alternativa frecuentemente recomendada (3) y esta con los otros tres casos hipotéticos a modo de análisis de alternativas. Se cuantifican los beneficios para los años 2005 y 2025, y luego los resultados son extrapolados al período 2015-2035 a una tasa social de interés del $4 \%$ para beneficios ambientales y de $9 \%$ para otros.

\section{Beneficios a ser cuantificados}

Hay cuatro beneficios que se atribuyen a los combustibles más limpios:

a. Ahorro de costos de salud debido a la exposición a un aire más limpio por menos dolencias relacionadas con la contaminación.

b. Ahorro de costos que no son de salud tales como menos corrosión de infraestructura, edificios, y de todo componente metálico. Se calcula como 16\% del beneficio (a) del párrafo anterior, basado en un promedio de seis ciudades en desarrollo (Lvosky et al., 2000).

c. Menores costos causados por reducir cambios del clima a nivel global por la reducción de emisiones de $\mathrm{CO}_{2}$, lo cual se valora en $\$ 20$ por tonelada de $\mathrm{CO}_{2}$ (Lvosky et al., 2000).

d. Cambios en precios y en productividad. Corresponde a mayores costos de producción del etanol o biodiésel, y menores precios ${ }^{40}$ de venta de los combustibles a gas, así como pequeñas diferencias de la productividad por combustible, que son un 3\% de mayor productividad del diésel sobre el biodiésel 5 y $2 \%$ de la gasolina sobre el etanol 7,8 .

\footnotetext{
40 Véase Tabla 4 en columnas bajo el subtítulo "Porcentaje consumido por autos, taxis y camiones".
} 


\section{Resultados DEL ANÁlisis de LA CONTAMINACIÓN AMBIENTAL}

\section{Cambios causados por pasos hacia combustibles más limpios}

La Tabla 4 presenta los cambios en el consumo de combustible causados por los cambios hacia combustibles más limpios, E7.8, B5, gas natural vehicular, y gas licuado de petróleo. Hay un pequeño cambio de usuarios de gasolina hacia gases mientras que desde diésel es más difícil. La situación se mantiene estable en tanto que los vehículos particulares consumen casi $90 \%$ de la gasolina o del E7.8, mientras que los buses de transporte

Tabla 4. Consumo de combustibles en el transporte de Lima y Callao $\left(^{*}\right)$

\begin{tabular}{|c|c|c|c|c|c|c|c|}
\hline antiguos & & & crecimiento & +limpios & & & crecimiento \\
\hline & 2005 & 2025 & $2025 / 2005$ & & 2005 & 2025 & $2025 / 2005$ \\
\hline Gasolina & 176 & 319 & $81 \%$ & E7.8 & 157 & 286 & $83 \%$ \\
\hline Diésel & 650 & 862 & $33 \%$ & B5 & 656 & 869 & $32 \%$ \\
\hline \multirow[t]{2}{*}{ Gases } & 81 & 135 & $67 \%$ & GLP & 98 & 163 & $66 \%$ \\
\hline & & & & GNV & 86 & 143 & $67 \%$ \\
\hline \multicolumn{8}{|c|}{ Porcentaje consumido por autos, taxis, y camiones } \\
\hline & 2005 & 2025 & precio $\$(* *)$ & & 2005 & 2025 & precio $\$(* *)$ \\
\hline Gasolina & $92 \%$ & $94 \%$ & 4.26 & E7.8 & $91 \%$ & $94 \%$ & 4.44 \\
\hline Diésel & $28 \%$ & $32 \%$ & 4.41 & B5 & $28 \%$ & $32 \%$ & 4.59 \\
\hline \multirow[t]{2}{*}{ Gases } & $100 \%$ & $100 \%$ & & GLP & $100 \%$ & $100 \%$ & 0.48 \\
\hline & & & & GNV & $100 \%$ & $100 \%$ & 0.49 \\
\hline \multicolumn{8}{|c|}{ Porcentaje consumido por buses de transporte público } \\
\hline Gasolina & $8 \%$ & $6 \%$ & & E7.8 & $9 \%$ & $6 \%$ & \\
\hline Diésel & $72 \%$ & $68 \%$ & & B5 & $72 \%$ & $68 \%$ & \\
\hline \multirow[t]{2}{*}{ Gases } & $0 \%$ & $0 \%$ & & GLP & $0 \%$ & $0 \%$ & \\
\hline & & & & GNV & $0 \%$ & $0 \%$ & \\
\hline
\end{tabular}

$\left.{ }^{*}\right)$ millones de galones para gasolina, diésel, E7.8, y B5

millones de litros para GLP

millones de metros cúbicos para GNV

$\left.{ }^{* *}\right)$ precios promedio al 2013

E7.8 Gasohol compuesto por 7,8\% etanol anhidro (de caña de azúcar) y el resto gasolina sin plomo.

B5 Biocombustible compuesto por 5\% biodiésel (de aceite de palma o aceite de soya) y el resto diésel de bajo azufre.

GLP Gas licuado de petróleo.

GNV Gas natural vehicular.

Gases Combustibles que pueden ser GLP o GNV en una proporción de consumo de mitad y mitad.

antiguos Caso de combustibles tradicionales como gasolina con plomo, diésel con azufre, y gases hasta un $7 \%$ de autos $y$ taxis.

+limpios Caso de combustibles más limpios como E7.8, B5, y combustibles de gases (gas licuado de petróleo y gas natural vehicular) hasta un $13 \%$ de los autos y taxis. 
público consumen casi $70 \%$ del diésel o del B5. Entre 2005 y 2025, el consumo de gasolina o de E7.8 crece mucho más que el consumo de diésel o de B5 porque la demanda de transporte en autos y taxis crece mucho más que la demanda de transporte público sobre buses.

La Tabla 5 presenta la cantidad de emisiones de contaminantes del transporte de Lima y Callao, en toneladas por año. En la columna "Reducción" se aprecian las reducciones de contaminantes debido a la conversión a combustibles más limpios. El material particulado (pm10) cae en -26\%, los óxidos de nitrógeno (nox) caen en -14\%, los óxidos de azufre (sox) caen en $-98 \%$, el plomo (pb) cae en $-100 \%$, y el dióxido de carbono $\left(\mathrm{CO}_{2}\right)$ cae en $-4.4 \%$. Estos enormes resultados son causados por pasos hacia el consumo de combustibles más limpios. Otro resultado es la reducción de la importancia de los buses del transporte público como contaminantes en -10 puntos porcentuales en material particulado (pm10), y en -58 puntos porcentuales en óxidos de azufre (sox) al cambiar de diésel a biodiésel sin sulfuros.

Table 5. Emisiones de contaminantes del transporte de Lima y Callao (tons por año)

\begin{tabular}{|c|c|c|c|c|c|c|c|}
\hline & \multicolumn{2}{|c|}{ Emisiones antiguos } & \multirow{2}{*}{$\begin{array}{c}\text { Crecim. } \\
2025 / 2005\end{array}$} & \multicolumn{2}{|c|}{ Emisiones + limpios } & \multirow{2}{*}{$\begin{array}{c}\text { Crecim. } \\
2025 / 2005\end{array}$} & \multirow[t]{2}{*}{ Reducción } \\
\hline & 2005 & 2025 & & 2005 & 2025 & & \\
\hline pm10 & 7,632 & 10,511 & $38 \%$ & 5,623 & 7,781 & $38 \%$ & $-26 \%$ \\
\hline Nox & 141,560 & 193,203 & $36 \%$ & 121,762 & 166,923 & $37 \%$ & $-14 \%$ \\
\hline Sox & 18,287 & 24,300 & $33 \%$ & 311 & 542 & $74 \%$ & $-98 \%$ \\
\hline $\mathrm{Pb}$ & 76 & 99 & $30 \%$ & - & - & & $-100 \%$ \\
\hline $\operatorname{co} 2\left(^{*}\right)$ & 9,858 & 14,191 & $44 \%$ & 9,422 & 13,568 & $44 \%$ & $-4.4 \%$ \\
\hline \multicolumn{8}{|c|}{ Porcentajes emitidos por autos, taxis, y camiones de carga } \\
\hline pm10 & $52 \%$ & $57 \%$ & & $63 \%$ & $67 \%$ & & \\
\hline Nox & $30 \%$ & $36 \%$ & & $34 \%$ & $40 \%$ & & \\
\hline Sox & $29 \%$ & $33 \%$ & & $87 \%$ & $91 \%$ & & \\
\hline $\mathrm{Pb}$ & $86 \%$ & $90 \%$ & & $0 \%$ & $0 \%$ & & \\
\hline co2 & $43 \%$ & $51 \%$ & & $44 \%$ & $51 \%$ & & \\
\hline \multicolumn{8}{|c|}{ Porcentajes emitidos por buses de transporte público } \\
\hline pm10 & $48 \%$ & $43 \%$ & & $37 \%$ & $33 \%$ & & \\
\hline Nox & $70 \%$ & $64 \%$ & & $66 \%$ & $60 \%$ & & \\
\hline Sox & $71 \%$ & $67 \%$ & & $13 \%$ & $9 \%$ & & \\
\hline $\mathrm{Pb}$ & $14 \%$ & $10 \%$ & & $0 \%$ & $0 \%$ & & \\
\hline $\operatorname{co} 2$ & $57 \%$ & $49 \%$ & & $56 \%$ & $49 \%$ & & \\
\hline pm10 & \multicolumn{7}{|c|}{ Material particulado de menos de 10 micras de diámetro. } \\
\hline Nox & \multicolumn{7}{|c|}{ Óxidos de nitrógeno. } \\
\hline Sox & \multicolumn{7}{|c|}{ Óxidos de azufre. } \\
\hline $\mathrm{Pb}$ & \multicolumn{7}{|c|}{ Plomo. } \\
\hline $\operatorname{co} 2$ & \multicolumn{7}{|c|}{ Dióxido de carbono o emisiones de gases de efecto invernadero. } \\
\hline
\end{tabular}

$\left(^{*}\right)$ miles de toneladas por año. 
Análisis de alternativas para reducir la contaminación causada por transporte urbano

La Tabla 6 muestra los beneficios de las alternativas para reducir la contaminación del transporte de Lima y Callao, en millones de dólares anuales así como en valor presente neto (vpn) para un período futuro de 20 años de 2015 a 2035.

La alternativa (1) de Combustibles más limpios ahorra costos de salud por 500 millones de dólares anuales, totalizando 8,5 billones de dólares en valor presente neto, que luego se reducen a 6,8 por incrementos de costos de producción de etanol y biodiésel.

La alternativa (2) de Inspección y mantenimiento ahorra costos de salud por 60 millones de dólares anuales; además, ahorra en productividad del combustible por motores más eficientes por 290 millones de dólares anuales, totalizando 3,8 billones de dólares en valor actual neto, que representa más de la mitad de los beneficios de la alternativa (1).

Tabla 6. Beneficios de alternativas de reducción de contaminación ambiental del transporte de Lima y Callao (millones de dólares)

\begin{tabular}{|c|c|c|c|c|c|c|c|c|}
\hline $\begin{array}{c}\text { Reducción de } \\
\text { costos en } \Rightarrow \\
\text { programas: }\end{array}$ & Año & $\begin{array}{l}\text { Costos de } \\
\text { salud }\end{array}$ & $\begin{array}{l}\text { Costos } \\
\text { salud, por } \\
\text { I/M }\end{array}$ & $\begin{array}{l}\text { Costos } \\
\text { no de } \\
\text { salud }\end{array}$ & $\begin{array}{c}\text { Costo de } \\
\text { emisiones } \\
\text { de } \mathrm{CO}_{2} \\
\end{array}$ & $\begin{array}{c}\text { Total } \\
\text { enfoque } \\
\text { gobierno }\end{array}$ & $\begin{array}{c}\text { Produc. y } \\
\text { precios }\end{array}$ & $\begin{array}{c}\text { Total } \\
\text { enfoque } \\
\text { usuario }\end{array}$ \\
\hline (1) comb. & 2015 & 445 & 24 & 75 & 10 & 555 & -156 & 399 \\
\hline \multirow[t]{2}{*}{+ limpios } & 2035 & 576 & 31 & 97 & 13 & 718 & -200 & 518 \\
\hline & $\underline{\mathrm{VPN}}$ & Z,147 & $\underline{388}$ & $\underline{833}$ & $\underline{165}$ & $\underline{8,532}$ & $\underline{-1,721}$ & $\underline{6,811}$ \\
\hline (2) inspección & 2015 & 55 & & 9 & 0.2 & 64 & 253 & 316 \\
\hline \multirow[t]{2}{*}{$\&$ mantenim. } & 2035 & 72 & & 12 & 1 & 84 & 336 & 420 \\
\hline & $\underline{\text { VPN }}$ & $\underline{887}$ & & 28 & 5 & 290 & $\underline{2,835}$ & $\underline{3,825}$ \\
\hline TOTAL & 2015 & 500 & 24 & 84 & 10 & 618 & 97 & 715 \\
\hline de política & 2035 & 649 & 31 & 109 & 14 & 803 & 136 & 939 \\
\hline actual $(1,2)$ & $\underline{\mathrm{VPN}}$ & $\underline{8,034}$ & $\underline{388}$ & 231 & $\underline{170}$ & 2,522 & $\underline{1,115}$ & $\underline{10,637}$ \\
\hline (3) reducir $1 \frac{1}{2}$ & 2015 & 231 & & 39 & 5 & 275 & 0 & 275 \\
\hline \multirow[t]{2}{*}{ flota de buses } & 2035 & 271 & & 46 & 6 & 323 & 0 & 323 \\
\hline & $\underline{\mathrm{VPN}}$ & $\underline{3,538}$ & & $\underline{412}$ & $\underline{82}$ & $\underline{4,032}$ & $\underline{0}$ & $\underline{4,032}$ \\
\hline (4) todo taxi & 2015 & 312 & & 50 & 20 & 381 & 469 & 850 \\
\hline \multirow[t]{2}{*}{ a GNV } & 2035 & 384 & & 61 & 25 & 471 & 601 & 1,072 \\
\hline & $\underline{\mathrm{VPN}}$ & $\underline{4,877}$ & & $\underline{541}$ & $\underline{316}$ & $\underline{5,733}$ & $\underline{5,158}$ & $\underline{10,892}$ \\
\hline (5) todo bus & 2015 & 239 & & 38 & 127 & 404 & 2,426 & 2,831 \\
\hline \multirow[t]{2}{*}{ a GNV } & 2035 & 290 & & 46 & 155 & 491 & 2,961 & 3,453 \\
\hline & $\underline{\text { VPN }}$ & $\underline{3,711}$ & & $\underline{412}$ & $\underline{1,971}$ & $\underline{6,094}$ & $\underline{26,134}$ & $\underline{32,228}$ \\
\hline (6) todo bus & 2015 & 7 & & 1 & 2 & 10 & 38 & 48 \\
\hline sobre arteria & 2035 & 8 & & 1 & 2 & 12 & 47 & 58 \\
\hline a GNV & $\underline{\text { VPN }}$ & $\underline{102}$ & & $\underline{11}$ & $\underline{31}$ & $\underline{145}$ & $\underline{412}$ & $\underline{556}$ \\
\hline TOTAL & 2015 & 551 & - & 88 & 147 & 786 & 2,895 & 3,681 \\
\hline de políticas & 2035 & 674 & - & 108 & 181 & 963 & 3,562 & 4,525 \\
\hline nuevas $(4,5)$ & $\underline{\text { VPN }}$ & $\underline{8,588}$ & $=$ & $\underline{953}$ & $\underline{2,286}$ & $\underline{11,827}$ & $\underline{31,292}$ & $\underline{43,120}$ \\
\hline
\end{tabular}




\begin{tabular}{lcccccccc}
\hline $\begin{array}{c}\text { Reducción de } \\
\text { costos en } \Rightarrow \\
\text { programas: }\end{array}$ & Año & $\begin{array}{c}\text { Costos de } \\
\text { salud }\end{array}$ & $\begin{array}{c}\text { Costos } \\
\text { salud, por } \\
\text { I/M }\end{array}$ & $\begin{array}{c}\text { Costos } \\
\text { no de } \\
\text { salud }\end{array}$ & $\begin{array}{c}\text { Costo de } \\
\text { emisiones } \\
\text { de CO } \text { CO }_{2}\end{array}$ & $\begin{array}{c}\text { Total } \\
\text { enfoque } \\
\text { gobierno }\end{array}$ & $\begin{array}{c}\text { Produc. y } \\
\text { precios }\end{array}$ & $\begin{array}{c}\text { Total } \\
\text { enfoque } \\
\text { usuario }\end{array}$ \\
\hline TOTAL & 2015 & 1,051 & 24 & 172 & 157 & 1,404 & 2,992 & 4,396 \\
todas alterna. & 2035 & 1,323 & 31 & 217 & 194 & 1,765 & 3,698 & 5,463 \\
(excepto (3)) & $\underline{\text { VPN }}$ & $\underline{16,621}$ & $\underline{388}$ & $\underline{1,884}$ & $\underline{2,456}$ & $\underline{21,349}$ & $\underline{32,407}$ & $\underline{53,756}$ \\
\hline
\end{tabular}

VPN

comb. + limpios

TOTAL de política actual

Todo bus sobre arteria a GNV

TOTAL de políticas nuevas

costos salud, por I/M
Valor presente neto durante el período 2015-2035.

Comprende gasohol E7.8 sin plomo, biodiésel B5 de bajo azufre, y combustibles de gases hasta $13 \%$ de los autos y taxis (gas licuado de petróleo GLP y gas natural vehicular GNV).

Incluye las políticas (1) y (2), cambio a combustibles más limpios y programa de Inspección y mantenimiento.

Cambiar todos los buses de transporte público que operan en una arterial de $24 \mathrm{~km}$. De largo a combustible GNV.

Cambiar todos los buses del transporte público y todos los taxis también a combustible GNV.

Ganancias adicionales en reducción de costos de salud por el cambio a combustibles más limpios, los cuales se obtienen solamente con la ayuda del programa de Inspección y mantenimiento.

La alternativa (3) de Reducción a la mitad de los buses del transporte público puede lograr ahorros de costos de salud por 250 millones de dólares anuales con un valor actual neto de 4.0 Billones de dólares. Los beneficios totales de alternativa (3) alcanzan tan solo el $38 \%$ de lo ya conseguido por las alternativas (1) y (2).

La alternativa (4) de Conversión de taxis a gas natural puede lograr ahorros en costos de salud por 350 millones de dólares anuales, y puede obtener ahorros por precios bajos de combustible por 550 millones de dólares anuales, para completar 10,9 billones de dólares en valor actual neto, $270 \%$ de los beneficios de la alternativa (3).

La alternativa (5) de Conversión de buses a gas natural puede lograr ahorros en costos de salud por 260 millones de dólares anuales, y puede obtener ahorros por precios bajos de combustibles por 2700 millones de dólares anuales, para completar 32,2 billones de dólares en valor actual neto, ocho veces los beneficios de la alternativa (3). Los menores beneficios por ahorros en costos de salud de la alternativa (5) de buses, con respecto a la alternativa (4) de taxis, se deben a que los buses ya están usando B5 de bajo contenido de azufre y más limpio.

La alternativa (6) Conversión de los buses que sirven una arterial de $24 \mathrm{~km}$. de longitud, a gas natural puede obtener ahorros en costos de salud por 7 millones de dólares anuales, y puede obtener ahorros por precios bajos de combustibles por 42 millones de dólares anuales, para completar 0,56 billones de dólares en valor presente neto, el 14\% de la alternativa (3) y en siete corredores se llega al $100 \%$ de la alternativa (3). 
Los beneficios totales de las políticas ya implementadas (1) y (2) ascienden a un valor presente neto de 10,6 billones de dólares, más de $160 \%$ del monto de las inversiones en la Refinería de Talara y en el Gasoducto del Sur. Pero, los beneficios de las políticas propuestas (4) y (5), ascienden a un valor presente neto de 43,0 billones de dólares, más de seis veces el valor de las inversiones públicas mencionadas.

\section{CONCLUSIONES Y RECOMENDACIONES SOBRE EL ANÁLISIS DE ALTERNATIVAS PARA REDUCIR LA CONTAMINACIÓN AMBIENTAL PROVENIENTE DEL TRANSPORTE URBANO}

Se han descrito las principales opciones existentes para reducir las emisiones de contaminantes provenientes del transporte urbano. La política de adoptar combustibles más limpios es la más recomendada a nivel mundial, y tiene requisitos técnicos de alto costo tales como refinamiento, gasoducto, e infraestructura de distribución. La política de inspección y mantenimiento produce la colaboración del propietario en mantener su vehículo. Política de reducir el número de buses de transporte público es la más frecuentemente recomendada, pero la más difícil de implementar porque ha tenido problemas que causan retrasos y porque originan costos sociales que falta presupuestar.

Primero, se ha encontrado que la alternativa (1) adopción de combustibles más limpios ha reducido enormemente la emisión de azufres Sox y plomos Pb, y además ha bajado moderadamente la emisión de material particulado pm10 y pm2.5, así como los nitrógenos Nox y los gases invernadero $\mathrm{CO}_{2}$. Con esta alternativa (1), el transporte público reduce su porcentaje de emisión de contaminantes en azufres Sox y en material particulado pm10. Sin embargo, se ha observado que el transporte público se ha convertido menos a combustibles a gas, lo que llama a cuantificar esta posibilidad en otras alternativas.

Luego, del análisis de alternativas se ha encontrado que los beneficios anuales son muy cuantiosos para todas las alternativas. Primero, las alternativas ya implementadas o en proceso de implementación: la alternativa (1) adopción de combustibles más limpios logra 500 millones de dólares anuales, la alternativa (2) inspección y mantenimiento logra 60 millones de dólares anuales, mientras que la alternativa (3) reducción del número de buses a la mitad logra solamente 259 millones de dólares, es decir, el $46 \%$ del beneficio anual de las alternativas (1) y (2) juntas. Segundo, las alternativas a ser potencialmente implementadas: la alternativa (4) conversión de los taxis a gas natural obtendría 350 millones de dólares anuales, la alternativa (5) conversión de buses a gas natural obtendría 260 millones de dólares anuales, y la alternativa (6) convertir todos los buses de una arterial donde se destina un carril para buses rápidos daría 7 millones de dólares anuales. Es decir, tanto las alternativas en implementación como las alternativas que podrían ser implementadas obtienen mayores beneficios anuales que la alternativa (3) de reducción del número de buses a la mitad. Se concluye entonces que el nivel de 
prioridad dado a la alternativa (3) debería bajarse y que el nivel de prioridad dado a las demás alternativas debería elevarse.

El análisis de alternativas indica que la alternativa (3) ha tenido una prioridad desproporcionadamente alta frente a otras alternativas que son más sencillas de implementar y que no tienen costos sociales, por lo que se recomienda reducir ostensiblemente el nivel de prioridad de la alternativa (3) Reducir la flota de buses del transporte público, cuando esta medida quiera usarse con fines de reducir la contaminación.

Por el otro lado, se recomienda fortalecer y seguir avanzando las alternativas (1) y (2) de conversión a combustibles más limpios con un buen sistema de inspección y mantenimiento. También se recomienda implementar las alternativas propuestas (4) conversión de taxis a gas natural y (5) de conversión de buses a gas natural vehicular u otro combustible limpio, y que se otorgue alta prioridad a las inversiones públicas de Refinería de Talara y del Gasoducto del Sur.

\section{RECOMENDACIONES FINALES SOBRE EL TRANSPORTE PÚBLICO DE BUSES VERSUS LA CONGESTIÓN Y LA CONTAMINACIÓN}

Se recomienda desestimar la medida de reducir la flota de buses de transporte público como medida para bajar la congestión por ser una medida inefectiva. Más bien, se recomienda estudiar otras medidas para reducir la congestión por ciertas horas, tales como los carriles-contra-sentido que aumentan la capacidad en la dirección-pico o semaforización que aumente el tiempo de luz verde en la dirección-pico, y construir infraestructura adicional en los accesos a los centros de Lima, Miraflores, o San Isidro, así como en aquellas zonas de escasa infraestructura como en La Molina.

También se recomienda aplicar la legislación de vías saturadas para reducir el número de buses en las horas-pico de 7 a.m., 8 a.m., 1 p.m. y 6 p.m., y en las que la influencia de los buses sea considerable, como a las 11 a.m., y también estudiar su utilización temporal en aquellas zonas de escasa infraestructura vial con el fin de garantizar la eficiencia de las vías. Aunque bien podría estudiarse combinar esta medida no solamente con la reducción de flujos de buses sino también con la reducción de flujos de autos, taxis y camiones si hubiera caminos adicionales que puedan ser utilizados.

También se recomienda reducir la prioridad de la medida de reducir el número de buses para bajar la contaminación porque tiene dificultades de implementación, falta presupuestar los costos sociales causados por esta política gubernamental, y hay mejores alternativas para el mismo objetivo. Más bien se recomienda priorizar la adopción de combustibles más limpios, apoyar el sistema de inspección y mantenimiento, y también, convertir a gas natural a taxis y a buses de transporte público. También se recomienda priorizar la inversión pública en la futura producción de la Refinería de Talara y el Gasoducto del Sur. 
Además, se recomienda implementar un limitado número de Sistemas de buses rápidos consistentes en segregar un carril de avenidas para uso exclusivo del transporte público por rutas de entre 20 a $24 \mathrm{~km}$. pues ejercen poca presión sobre la congestión y si se utilizaran buses a gas, daría beneficios adicionales por 7 millones de dólares anuales y otros 53 millones de dólares anuales para el operador debido a la diferencia de precios de los combustibles.

\section{REFERENCIAS}

Andreoli, C. y S.P. de Souza (2006). Sugarcane: The Best Alternative for Converting Solar and Fossil Energy into Ethanol. Study presented at the Conference of Agri Energy International, 11-13 December. Londrina, PR, Brazil, Economy \& Energy IX-\#59.

Ardila, Arturo (2008). Limitation of Competition in and for the Public Transportation Market in Developing Countries. Lessons from Latin American Cities. Transportation Research Record: Journal of the Transportation Research Board, No. 2048, Transportation Research Board of the National Academies, Washington, D.C., 2008, pp. 8-15.

Ardila, Arturo y Daniel A. Rodriguez (2000). How Chaos Does Not Destroy Ridership. Operations of an Exclusive Busway Carrying More than 35,000 Passengers per Hour per Direction. TRR, 1726, 1-7.

Armann, D. y N. Sieber (2005). Transport in Developing Countries: Renewable Energy versus Energy Reduction. Transport Reviews, 25(6), 719-738.

Armstrong-Wright, Alan y Sebastien Thiriez (1987). Bus Services. Reducing Costs, Raising Standards. Washington, D.C.: The World Bank.

Autoridad Autónoma del Tren Eléctrico (2002). Manual de Toma de Datos de Tiempos de Viaje $y$ de Estimación de Funciones Volumen-Demora. Lima: Gerencia de Desarrollo para el Estudio del Proyecto del Sistema MetroBus.

Bai, S., Y.C. Chiu y D.A. Niemeier (2007). A Comparative Analysis of using Trip-Based versus Link-Based Traffic Data for Regional Mobile Source Emissions Estimation. Atmospheric Environment, 41, 7512-7523.

Barth, M., F. An, J. Norbeck y M. Ross (1996). Modal Emissions Modeling: A Physical Approach. Journal of the Transportation Research Board, Transportation Research Record 1520, pp.81-88.

Barth, M., F. An, T. Younglove, G. Scora, C. Levine, M. Ross, T. Wenzel (2000). Development of a Comprehensive Modal Emissions Model, NCHRP web-only document 122, TRB, California.

Bustamante, H.G. (2013). Barreras para el desarrollo de la bioenergía. Tomo VIII de Matriz Energética en el Perú y Energías Renovables. Lima-Perú.

Cesar, H. (2002). Improving Air Quality in Metropolitan Mexico City: An Economic Evaluation. Policy Research Working Paper 2785. Washington, D.C.: The World Bank.

Chatterjee, A.; Miller, T.L; Philput, J.W.; Wholly, Jr., T.F.; Guensler, R.; Hartgen, D.; Mergiotta, R.A. y Stopher, P.R. (1997). Improving Transportation Data for Mobile Source Emission Estimates NCHRP Report 394. TRB.

CONAM (2005). Guía de Estimación del Costo-Beneficio de Medidas para el Saneamiento Atmosférico by CONAM and Kroeger, T. et al. Lima, Perú. 
Davis, N., J. Lents, M. Osses, N. Nikkila y M. Barth (2005). Development and Application of an International Vehicle Emissions Model. Journal of the Transportation Research Board, Transportation Research Record 1939, pp.157-165.

Defensoría del Pueblo (2008). El Transporte Urbano en Lima Metropolitana: Un desafío en defensa de la vida. Informe Defensorial 137 de la Defensoría del Pueblo, Lima, Perú.

Delucchi, M.A. (2001). A Lifecycle Emissions Analysis: Urban Air Pollutants and GreenhouseGases from Petroleum, Natural Gas, LPG, and Other Fuels for Highway Vehicles, Forklifts, and Household Heating in the U.S. World Resource Review 13(1), 25-51.

Diaz, R.B. y Donald C. Schneck (2000). Bus Rapid Transit Technologies in the Americas. An Overview. TRR, 1731, 3-9.

Dowling, R., R. Ireson, A. Skabardonis, D. Gillen y P. Stopher, P. (2005). Predicting Air Quality Effects of Traffic-Flow Improvements: Final Report and User's Guide NCHRP Report 535. TRB. Washington, D.C.

Estache, Antonio y Andres Gomez-Lobo (2004). The Limits of Competition in Urban Bus Services in Developing Countries. World Bank Policy Research Working Paper 3207.

Gardner, G., P.R. Cornwell y J.A. Cracknell (1991). The Performance of Busway Transit in Developing Cities. Research Report 329. TRRL,

Gobierno Peruano (2001). Revisión Técnica de Vehículos Limites Máximos Permisibles de Emisiones preparado por Fundación Suiza de Cooperación para el Desarrollo Técnico para el Comité de Gestión de la Iniciativa de Aire Limpio para Lima y Callao, Lima, Perú.

Gobierno Peruano (2004). Perspectivas del Medio Ambiente Urbano: GEO Lima y Callao preparado por PNUMA, CONAM, Municipalidad Metropolitana de Lima, MPC, GEA, Financiado por Gobierno de Noruega, Lima, Perú.

Gwilliam, K. (2002). Cities on the Move: A World Bank Urban Transport Strategy Review. Washington, D.C.: The World Bank.

Gwilliam, K. y R. Scurfield (1996). Competition in Public Road Passenger Transport. Paper TWU-39. Washington, D.C.: The World Bank

Gwilliam, K., M. Kojima y T. Johnson. (2004) Reducing Air Pollution from Urban Transport. Washington, D.C.: The World Bank.

Heil, M. y S. Pargal (1998). Reducing Air Pollution from Urban Passenger Transport: A Framework for Policy Analysis Policy Research Working Paper. The World Bank, a product of Infrastructure and Environment Development Research Group. Washington, D.C.

Hook, Walter (2005). Institutional and Regulatory Options for Bus Rapid Transit in Developing Countries. Lessons from International Experience. TRR, 1939, 184-191.

Jacobs, G.D., P.R. Fouracre y D.A.C. Maunder (1982). Public Transport in Third World Cities. The Journal of the Institution of Highway Engineers. March.

JHK \& A. (c. 1978). Design of Urban Streets: Training Course for Operating Agencies Prepared for the Federal Highway Administration.

Kojima, M. (2001). Breathing Clean: Considering the Switch to Natural Gas Buses. World Bank Technical Paper \# 516. Washington, D.C.: The World Bank.

Kojima, M. y M. Lovei (2001). Urban Quality Management: Coordinating Transport, Environmental and Energy Policies in Developing Countries World Bank Technical Paper 508. Washington, D.C.: The World Bank.

Kokaz, K. y P. Rogers (2002). Urban Transportation Planning for Air Quality Management: Case Study in Delhi, India, of Role of Social and Economic Costs in Welfare Maximization 
of Mobility Choice. Journal of the Transportation Research Board, Transportation Research Record 1817, 42-49.

Kroger, T. (2005). Descontaminación Atmosférica Metropolitana: Beneficios en la Salud y Costos Lima.

Lents, J., N. Davis, N. Nikkila y M. Osses (2004). Lima Vehicle Activity Study. ISSRC.

Lvovsky, K., G. Hughes, D. Maddison, M. Ostro y D. Pearce (2000). Environmental Costs of Fossil Fuels: A Rapid Assessment Method with Application to Six Cities Environment Department papers No. 78. Washington, D.C.: The World Bank.

Malbrán, R., H. (1998). Transporte público bajo gestión privada: la experiencia de licitación de vías en Santiago de Chile. En Conam-Peru (ed.), Transporte urbano y ambiente: bases para una politica ambiental en el transporte urbano (pp. 159-167). Lima: Consejo Nacional del Ambiente.

Martinez, M.J. y J. Cornejo (2003). Value of the Facilities and Attributes of New Heavy Rail and Bus Rapid Transit Projects in a Developing City. The Case of Lima, Peru. TRR, 1835, 50-58.

Martinez, Manuel J. (2009). Calculation of Benefits of Advanced Integrated Rail Service. Application of Intelligent Transportation Systems to the Developing City of Lima, Peru. Transportation Research Record, Journal of the Transportation Research Board, 2112, 26-33. Washington, D.C.: Transportation Research Board of the National Academies.

Martinez, Manuel José (2008). Los desafíos confrontados por los proyectos convencionales de transporte y el potencial de los sistemas inteligentes de transporte para una ciudad en desarrollo, Lima, Perú. ECIPerú, 4(1), 14-17. Recuperado de https://revistaeciperu.files. wordpress.com/2016/07/eci2007vvol4num1.pdf

Ministerio de Transportes y Comunicaciones (1999). Vitrina, Asistencia Técnica en Estudios de Transporte Urbano para el Área Metropolitana de Lima y Callao. Informe Final. Informe de Resultados. Financiado por el World Bank.

Ministerio de Transportes y Comunicaciones (2005). The Master Plan for Lima and Callao Metropolitan Area Urban Transportation in the Republic of Peru.

Municipalidad Metropolitana de Lima (2005). Estudio de Linea Base Ambiental COSAC I Informe Final Preparado por Deuman International y Walsh Ingenieros y Científicos Consultores para Protransporte, Lima.

Municipalidad Metropolitana de Lima-ProTransporte (2002). Estudio de los impactos ambientales en el área de influencia directa del Corredor Segregado de Alta Capacidad COSAC / PTUL. Preparado por Getinsa / Taryet. Funded by the IAD.

Municipalidad Metropolitana de Lima-ProTransporte (2011). Perfil de Proyecto Construcción Corredor vial de Transporte Público Masivo Este-Oeste Carretera Central-Av. Grau-Av. Venezuela, Provincia de Lima.

Ostro, B. (1994). Estimating the Health Effects of Air Pollutants: A Method with an Application to Jakarta. Policy Research Working Paper 1301. Washington, D.C.: The World Bank.

Quispe Cornejo, Silvia (2007). Geografía del transporte vial en Lima Metropolitana y su impacto vial territorial. Revista Investigaciones Sociales, XI(19), 151-171. Recuperado de revistainvestigaciones.unmsm.edu.pe/index.php/ sociales/article/newfile/8050/17022.

Rogers, P., S. Srinivasan y K. Kokaz (2001). Transportation and Environment: Problems in Delhi and Beijing Internet Video at World Bank Seminar Brown Bag Meeting.

Shalizi, Z. y J.C. Carbajo (1994). Transport-Related Air Pollution Strategies: What Lessons For Developing Countries Report TWU 14. Washington, D.C.: The World Bank, 
Environmentally Sustainable Development, Transportation, Water \& Urban Development Department, Transport Division.

Sheehan, J., V. Camobreco, J. Duffield, M. Grabosky y H. Shapouri (1998). Life Cycle Inventory of Biodiesel and Petroleum Diesel for Use in an Urban Bus. Golden, CO: National Renewable Energy Laboratory.

Tapia, J. y C. Zegrass (1998). "Transporte y cambio climático: resultados del estudio de opciones de mitigacion” Transporte Urbano y Ambiente, ed. by CONAM (pp. 91-96)

The World Bank (1975). Urban transport. Sector policy paper. Washington, D.C.: World Bank Office.

The World Bank (1996). Sustainable Transport: Priorities for Policy Reform. Washington, D.C.: Transportation, Water and Urban Development Division.

The World Bank (2001). Vehicular Air Pollution: Setting Priorities. South Asia Urban Air Quality Management Briefing Note No. 1, Washington, D.C.: The World Bank.

Thomson, I. y A. Bull (2001). La congestión del tránsito urbano: causas y consecuencias económicas y sociales. Serie Recursos Naturales e Infraestructura 25. Santiago de Chile: CEPAL.

US-DOT-FHWA (1994). Development and Testing of Dynamic Traffic Assignment and Simulation Procedures for ATIS/ATMS Applications. Austin, TX: Center for Transportation Research, the University of Texas at Austin.

US-DOT-FHWA (2007). Dynasmart-P User's Guide.

USDOT (1979). Fundamentals of Air Quality: Implementation Package 76-5. Reprinted. Prepared by Greiner E.S.Inc for the FHWA Baltimore, Maryland.

Wang, M., J. Han, J. Dunn, H. Cai y A. Elgawaing. (2012). Well-to-Wheels Energy Use and Greenhouse Gas Emissions of Ethanol from Corn, Sugarcane, Corn Stover, Switchgrass, and Miscinthus. Environmental Research Letter, 7045905. 